\title{
Activation of $\beta$-Catenin Cooperates with Loss of Pten to Drive AR-Independent Castration-Resistant Prostate
}

\section{Cancer Ac}

Rachana Patel', Elspeth A. Brzezinska', Peter Repiscak ${ }^{1,2}$, Imran Ahmad $^{1,2}$, Ernest Mui, ${ }^{1,2}$, Meiling Gao', Arnaud Blomme', Victoria Harle ${ }^{1,2}$, Ee Hong Tan', Gaurav Malviya', Agata Mrowinska', Carolyn J. Loveridge ${ }^{1,2}$, Linda K. Rushworth, ${ }^{1,2}$, Joanne Edwards' ${ }^{2}$, Chara Ntala', Colin Nixon', Ann Hedley ${ }^{1}$, Gillian Mackay', Saverio Tardito', Owen J. Sansom ${ }^{1,2}$, and Hing Y. Leung ${ }^{1,2}$

\section{ABSTRACT}

Inhibition of the androgen receptor (AR) is the main strategy to treat advanced prostate cancers. AR-independent treatmentresistant prostate cancer is a major unresolved clinical problem. Patients with prostate cancer with alterations in canonical WNT pathway genes, which lead to $\beta$-catenin activation, are refractory to AR-targeted therapies. Here, using clinically relevant murine prostate cancer models, we investigated the significance of $\beta$-catenin activation in prostate cancer progression and treatment resistance. $\beta$-Catenin activation, independent of the cell of origin, cooperated with Pten loss to drive AR-independent castrationresistant prostate cancer. Prostate tumors with $\beta$-catenin activation relied on the noncanonical WNT ligand WNT5a for sustained

\section{Introduction}

Androgen deprivation therapy (ADT) that blocks androgen receptor (AR) activity is the preferred standard-of-care treatment for patients with advanced prostate cancer $(1,2)$. Although ADT offers palliative benefits, most patients eventually progress to a lethal treatment-resistant stage, referred to as castration-resistant prostate cancer (CRPC). Although reactivation of AR signaling occurs in a majority of CRPC cases, the proportion of AR-independent prostate cancer is increasing in the emerging CRPC (3).

Genomic alterations and therapy-induced plasticity may cause the emergence of AR-independent CRPC. Therefore, understanding the functional consequences of clinically observed genomic alterations in the emergence of CRPC may facilitate the development of appropriate treatment strategies, especially for AR-independent tumors. One such pathway implicated in CRPC is WNT/B-catenin signaling (4-6). BCatenin is an effector of the WNT signaling that regulates multiple biological processes (7). The localization and stability of ß-catenin

${ }^{1}$ Cancer Research UK Beatson Institute, Glasgow, Scotland, United Kingdom. ${ }^{2}$ Institute of Cancer Sciences, Glasgow, Scotland, United Kingdom.

Note: Supplementary data for this article are available at Cancer Research Online (http://cancerres.aacrjournals.org/).

R. Patel and E.A. Brzezinska contributed equally to this article.

Corresponding Authors: Hing Y. Leung, CRUK Beatson Institute, Garscube Estate, Switchback Road, Glasgow, Scotland G61 1BD, United Kingdom. Phone: 0141-330-3658; Fax: 0141-942-6521; E-mail:

h.Leung@beatson.gla.ac.uk; and Rachana Patel, rachanapatel@gmail.com

Cancer Res 2020;80:576-90

doi: 10.1158/0008-5472.CAN-19-1684

(C)2019 American Association for Cancer Research. growth. WNT5a repressed AR expression and maintained the expression of c-Myc, an oncogenic effector of $\beta$-catenin activation, by mediating nuclear localization of NFאBp65 and $\beta$-catenin. Overall, WNT/ $\beta$-catenin and AR signaling are reciprocally inhibited. Therefore, inhibiting $\mathrm{WNT} / \beta$-catenin signaling by limiting WNT secretion in concert with AR inhibition may be useful for treating prostate cancers with alterations in WNT pathway genes.

Significance: Targeting of both AR and WNT/ $\beta$-catenin signaling may be required to treat prostate cancers that exhibit alterations of the WNT pathway.

protein are tightly regulated to maintain epithelial homeostasis. Oncogenic activation of $B$-catenin results in a stable protein that translocates to nucleus and functions as a transcriptional coactivator of $\mathrm{T}$-cell factor (TCF) family transcription factors leading to transcription of target genes, such as MYC, to govern growth and metabolism (8). WNT ligands, such as WNT5A, may govern cancer progression through both canonical $B$-catenin activation and noncanonical signaling $(9,10)$.

The functional relevance of oncogenic activation of $B$-catenin in prostate cancer progression and treatment-resistance is not clear (11-13). Here, using preclinical murine and human prostate cancer models, we have investigated the functional impact of oncogenic B-catenin activation and WNT signaling in prostate cancer progression and defined the underlining mechanisms of treatment resistance. We show that $B$-catenin activation mediates ARindependent CRPC through WNT5a. Overall, the WNT/ß-catenin and AR signaling pathways exhibit mutual antagonism and dual targeting of these pathways may be required to treat patients with WNT pathway alterations.

\section{Materials and Methods}

\section{Study approvals}

All animal experiments were approved by the Animal Care and Use Committee at the University of Glasgow with ethical approval under the revised Animal (Scientific Procedures) Act 1986 and the EU Directive 2010/63/EU (PPL P5EE22AEE). For clinical samples, ethical approval was obtained from the West of Scotland Research Ethics Committed (05/S0704/94).

Human prostate cancer samples and analyses

B-Catenin IHC was performed in prostate cancer tissue microarray (TMA) generated using diagnostic biopsies from 33 patients with 
suitable cores for analyses. Twenty-seven patients of this TMA cohort progressed to develop CRPC. For each stained section, the staining intensity and percentage of cells stained were scored accounting for membranous, cytosolic, and nuclear staining. Semiquantitative classification of staining intensity, the histoscore was calculated as previously described (14). Based on the cumulative scores, the analyzed cores were segregated into cancers with high (above median IHC score) or low (below median IHC score) levels of $\beta$-catenin for further survival analyses. For survival analyses, 27 patients that progressed to CRPC were selected and based on IHC histoscore divided into patients with high and low B-catenin. Retrospective PTEN IHC scores (14) of ADT-treated patients $(n=33)$ were used for correlation analyses. Survival analysis based on low or high $ß$-catenin staining was carried out in subset of ADT-treated patients with low (below median IHC score; $n=17$ ) or high (above median IHC score; $n=16$ ) levels of PTEN.

The genomic alterations in WNT pathway were investigated in The Cancer Genome Atlas (TCGA) provisional dataset for prostate cancer in patients treated with postoperative targeted therapy (15). The clinical information on the patients that received targeted therapy $(n=252)$ were obtained from the cBio Portal Genomic Portal $(16,17)$. The patients were segregated into patients with and without alterations in WNT pathway genes, which may lead to $B$-catenin activation, to perform Kaplan-Meier analyses for progression-free survival following cancer-specific standard-of-care treatments. The patients that did not receive any postoperative adjuvant targeted or pharmacologic treatments were not used for analyses. Prostate adenocarcinoma TCGA provisional (15) dataset was used to determine the significance of WNT expression $(z$-score $>1.4)$ on progression-free survival. The Kaplan-Meier survival curves on the extracted data were plotted using GraphPad Prism.

Mice

The ARR2 Probasin-Cre (Pb-Cre; ref. 18), Pten flox (19), Ctnnb1 $1^{(\mathrm{ex} 3) \Delta /+}(20), N k x 3.1 C r e^{\mathrm{ERT} 2}(21)$, and $R F P /+(22)$ mice have been previously described. All mice in this study were on a mixed background and were genotyped by Transnetyx using PCR analysis of ear notch tissue. The male littermates with only Cre expression or without any genetic alterations were used as the wild-type (WT) controls. Genotype-specific male mice were handled and aged until experimental time-points or ethically approved clinical endpoints. The expression of Cre recombinase in Nkx3-1 ${ }^{\text {CreERT2 }}$ mice was induced at 12 weeks of age with $160 \mathrm{mg} / \mathrm{kg}$ tamoxifen, administered by i.p. injection 4 times over an 11-day period. The commercially available immunocompromised CD-1 Nudes 6- to 8-week-old male mice were used for orthograft tumor experiments as previously described (23). For orthograft experiments, approximately 5 million murine prostate cancer cells were injected in one of the anterior prostate lobes of male CD-1 Nude mice. Mice were gavaged with LGK974 $(1.25 \mathrm{mg} / \mathrm{kg})$ and its vehicle $(0.5 \%$ methyl cellulose $/ 0.5 \%$ Tween 80$)$ once daily for 1 -month post-ADT treatment. The ARRIVE guidelines were used to design and execute experiments.

\section{Cell lines}

Human CRPC cell line, C4-2, was purchased from the ATCC, and PC-3M cells are metastasis-derived variant of human prostate cancer PC3 cells (24). Cell lines were mycoplasma negative and authenticated by LGC standards. Murine prostate cancer cell lines (P1, P2, CP1, and CP2) were generated for this study. P1 and P2 cell lines (RRID: CVCL_VQ82; RRID:CVCL_VQ83) were derived from prostate tumors from Pten $^{p c-/-}$ mice at 14 and 13 months, respectively. CP1 and CP2 cell lines (RRID:CVCL_VQ84; RRID:CVCL_VQ85) were derived from prostate tumors from $C$ tnn $b 1^{p c(e \times 3) \Delta /+} \operatorname{Pten}^{p c+/-}$ mice at 6 and 8 months, respectively. P1, P2, CP1, and CP2 cell lines were derived using an adaptation of method published before (25).

\section{Immunostaining, immunofluorescence, immunoblotting, and immunoprecipitation}

IHC, immunofluorescence, and immunoblotting were performed as previously described $(23,26)$. Immunoprecipitation was carried out using Dynabeads guidelines with lysates from freshly harvested prostate tumors (scale bar, $100 \mu \mathrm{m}$ ).

\section{RNA sequencing}

The library preparation and sequencing on extracted RNA was carried out by BGI (Beijing). Expression counts were further processed and analyzed in the R environment (version 3.2.3; GSE136080, GSE136082, and GSE136084).

\section{Chromatin immunoprecipitation assay}

Minced frozen prostate tissue was fixed using $1 \%$ formaldehyde for 15 minutes and quenched with Glycine $(125 \mathrm{mmol} / \mathrm{L})$ at room temperature for 5 minutes. Tissues were homogenized, nuclei isolated, and lysed. Nuclear lysates were sonicated using the Bioruptor sonicator on high setting 30 -second on/30-second off for 25 minutes $(2 \times 10$ minutes $+1 \times 5$ minutes). Precleared chromatin samples using IgG were used for chromatin immunoprecipitation (ChIP) assay with AR or HA (ChIP control) antibody with protein G-agarose suspension. After ChIP at $4{ }^{\circ} \mathrm{C}$ with rotation overnight, the pellet was washed and DNA isolated and dissolved for sequencing, which was carried out at BGI or quantitative PCR analysis. Regions of AR occupancy were determined using USeq Version 8.6.6 and MACS Version 1.4.1 (GSE136077).

\section{Gene set enrichment analyses and cluster analyses}

Clustering analysis was performed on the $\log _{2}$-transformed DESeq2-normalized counts for genes that are significantly differentially expressed between a genotype and the respective control and that are present in all of the comparisons. The significance was defined as the pi score larger than $1.3(\pi>=1.3)$, where $\pi=-\log _{10}($ p.adj $) \times$ abs $\left(\log _{2} \mathrm{FC}\right)$. For clustering, hierarchical clustering from R stats package was used with correlation distance and average agglomeration method. Gene set enrichment analysis (GSEA) with Hallmarks gene set collection from Molecular Signature Database was carried out using GSEAPreranked algorithm with genes from RNA sequencing (RNA-seq) differential expression analysis ranked according to the pi score (defined above).

\section{Murine model signatures}

Genes significantly (p.adj $<0.05$ and $\operatorname{abs}\left(\log _{2} \mathrm{FC}\right)>1$ ) upregulated or downregulated in prostate tumors with $B$-catenin activation compared with Pten deletion common in both genetically engineered mouse model and orthografts were used to generate $ß$-catenin Up and Down genesets, respectively. Human version of genesets was generated by converting genes from mouse ensembl IDs to human gene symbols using biomaRt (v2.38.0) with ensembl version 92 (http://apr2018.archive.ensembl.org) and removing duplicated gene symbols or gene symbols not mapped (https://github.com/prepiscak/beta_catenin_article).

\section{Seahorse bioanalyzer assay}

The Seahorse XFe96 Analyzer and Seahorse XF Cell Mito Stress Test Kit were used to measure the oxygen consumption rates (OCR) and 
extracellular acidification rates (ECAR) accordingly to the manufacturer's instructions. Total final readings were normalized to protein content.

\section{Transfections}

All siRNA and plasmid transfections in CP and PC3M cells were done using nucleofection (Lonza-kit V). Active WNT5A-V5 was a gift from Xi He (Addgene plasmid \# 43813). C4-2 cells were transfected using nucleofection (Lonza-kit R).

\section{Statistical analysis}

GraphPad Prism 7 was used for statistical analyses. Each data-point represents a tumor and data shown as mean $\pm \mathrm{SD}$. For significance, * or \#, $P<0.05$. For Kaplan-Meier survival analyses, the Logrank (Mantel-Cox) test was used.

Detailed methods are available in the Supplementary Information.

\section{Results}

\section{B-Catenin activation cooperates with Pten loss to mediate prostate cancer progression}

Patients with prostate cancer with alterations in the canonical WNT pathway genes (APC, CTNNB1, RSPO2, RNF43, and ZNRF3) leading to $B$-catenin activation (4) have a significantly poorer prognosis and survival compared with patients without any alterations (Fig. 1A and B; Supplementary Fig. S1A). Patients with prostate cancer with CTNNB1 (B-catenin) alterations relapse sooner than patients without alterations (Fig. 1C; Supplementary Fig. S1B). Although genomic CTNNB1 alterations occur in various solid cancers, they are significantly associated with poor prognosis only in prostate cancer where the standard-of-care treatment is AR-targeted therapies (Fig. 1D; Supplementary Fig. S1C). The reason for a difference in the observed clinical impact is unclear but may suggest that prostate cancers with CTNNB1 alterations are refractory to AR-targeted therapies. To investigate the clinical relevance of $B$-catenin, we studied the $B$-catenin expression in diagnostic prostate cancer biopsies by IHC (Fig. 1E). Patients with high levels of tumoral $B$-catenin who have progressed to CRPC have significantly reduced survival compared with those with low $B$-catenin levels (Fig. 1F).

PTEN levels showed a modest but significant inverse correlation with ß-catenin levels (Fig. 1G). Only in ADT-treated patients with low tumoral PTEN, patients with high $ß$-catenin levels have significantly poorer survival than those with low $ß$-catenin levels (Fig. 1H; Supplementary Fig. S1D). Forty-three percent of primary prostate adenocarcinomas and metastases with CTNNB1 mutations also have PTEN loss (Supplementary Fig. S1E; ref. 6). These data may suggest cooperation between oncogenic $B$-catenin activation and PTEN loss in prostate cancer progression.

To investigate the functional cooperation between $B$-catenin activation and Pten loss, we used a conditional Cre-loxP system driven by Probasin-Cre expressed in both basal and luminal cells of the murine prostatic epithelium. The $C \operatorname{tnn} b 1^{(e \times 3) \Delta /+}$-mutant mouse model, where a single allele of Ctnnb1 gene has loxP site flanking the exon 3, was used to achieve constitutive activation of B-catenin (20). The survival of mice with prostate-specific activation of $B$-catenin and heterozygous or homozygous loss of Pten ${ }^{(C t n n b 1} 1^{p c(e x 3) \Delta /+}$ Pten $^{p c+/-}$ or Ctnnb $1^{p c(e x 3) \Delta /+}$ Pten $^{p c-/-}$, respectively) was significantly shorter compared with mice with either ß-catenin activation $\left(\operatorname{Ctnnb1}^{p c(e \times 3) \Delta /+}\right)$, heterozygous $\left(\right.$ Pten $\left.^{p c+/-}\right)$, or homozygous $\left(\right.$ Pten $\left.^{p^{c-l}-}\right)$ Pten loss alone (Fig. 1I). Highlighting the functional cooperation between Pten and $B$-catenin, the survival of mice with $ß$-catenin activation and Pten deletion was significantly shorter than mice with $ß$-catenin activation and heterozygous loss of Pten (Fig. 1I). Although the heterozygous, Pten loss resulted in prostatic intraepithelial neoplasia (PIN) lesions, mice from the other four genotypes developed prostate cancer. At 6 months, the prostate tumor burden was significantly higher in double-mutant $C_{\text {tnnb1 }}{ }^{p(\text { ex } 3) \Delta /+} \mathrm{Pten}^{p c+/-}$ mice than in Ctnnbl-mutant alone mice (Supplementary Fig. S1F). Importantly, at clinical endpoint, Pten levels were comparable between Ctnnb1-mutant tumors with either heterozygous or homozygous Pten deletion (Supplementary Fig. S1G-S1H). Mice with Ctnnb1-mutant prostate tumors with complete Pten deletion had a significantly higher tumor burden compared with mice with Ctnnb1-mutant, Pten deletion or Ctnnb1 mutant with Pten haploinsufficiency (Fig. 1J). Thus, the Ctnnb1activating mutation cooperates with Pten copy-number alterations to accelerate prostate cancer progression.

\section{B-Catenin activation drives AR-independent metastatic CRPC}

We next investigated the effects of ADT, given in the form of castration, on murine tumors with $\beta$-catenin activation. ADT in $C_{\text {tnnb }} 1^{p c(e x 3) \Delta /+}$ Pten $^{p c+/-}$ or Ctnnb $1^{p c(e x 3) \Delta /+}$ mice did not significantly alter the survival or tumor burden (Fig. 2A-D). Similar to clinical prostate cancer, Ctnnb1-mutant murine tumors appeared to be refractory to ADT. Based on AR and B-catenin staining, we observed two patterns in hormone-naïv (HN) $C_{t n n b} 1^{p c(e x 3) \Delta /+}$ Pten $^{p c+/-}$ prostate tumors, one with the glandular areas exhibiting prominent $A R$ staining with no nuclear ß-catenin (red highlight, Fig. 2E), contrasting with poorly differentiated areas (lacking the glandular structures) with prominent nuclear B-catenin staining and little detectable AR (black highlight, Fig. 2E). Compared with $\mathrm{HN}$ tumors, the CRPC tumors in Ctnnb1 $1^{p c(e \times 3) \Delta /+} \mathrm{Pten}^{p c+/-}$ and Ctnnb1 $1^{p c(e \times 3) \Delta /+}$ mice had little detectable AR (Fig. 2E; Supplementary Fig. S2A and S2B). At clinical endpoint, more than half of the Ctnnb1 ${ }^{p c(e x 3) \Delta /+} P_{t e n}{ }^{p c+/-}$ mice and a quarter of $C t n n b 1^{p c(e \times 3) \Delta /+}$ mice developed visceral metastases (Supplementary Fig. S2C and S2D). Lung metastases were also evident in more than half of the mock and ADT-treated $C_{\text {Ctnnb }}{ }^{p c(e x 3) \Delta /+}$ Pten $^{p c+/-}$ mice (Supplementary Fig. S2E). HN and CRPC lung metastases had prominent nuclear B-catenin, and CRPC lung metastases were deficient for AR (Supplementary Fig. S2F). Overall, $B$-catenin activation drives AR-independent metastatic CRPC (mCRPC).

Prostate cancer can arise from both the AR-positive luminal and AR-negative basal cells within the prostatic epithelium. Because Probasin-Cre-mediated recombinations occur in both luminal and basal cells of murine prostatic epithelium, AR-deficient mCRPC observed in the Ctnnb $1^{p c(e x 3) \Delta /+}$ Pten $^{p c+/-}$ mice may reflect selective survival or expansion of AR-negative basal cells. Hence, to investigate the functional role of Ctnnb1 mutation in mediating castration resistance in AR-proficient cells, we applied the tamoxifeninducible Nkx3-1CreERT2 Cre-loxP system to induce genetic recombination in the luminal prostatic epithelial cells (Supplementary Fig. S3A; ref. 21). Using red fluorescent protein expression reporter, we established an induction protocol to achieve $80 \%$ to $90 \%$ recombination as indicated by nuclear ß-catenin staining in Ctnnb1-mutant prostates (Supplementary Fig. S3B-S3D).

The survival of mice with prostate luminal cell-specific activation of $B$-catenin with the heterozygous or homozygous loss of Pten (namely Ctnnb1 $1^{\text {Nkx3-1CreERT2(ex3) } /++}$ Pten $^{\text {Nkx3-1CreERT2+/- }}$ or

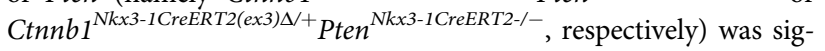
nificantly lower compared with mice with either ß-catenin activation $\left(C_{t n n b} 1^{\text {Nkx3-1CreERT2(ex3) L/+ }}\right)$, heterozygous $\left(\right.$ Pten $\left.^{\text {Nkx3-1CreERT2+/- }}\right)$, or 
$\beta$-Catenin Activation Induces AR-Independent CRPC

A

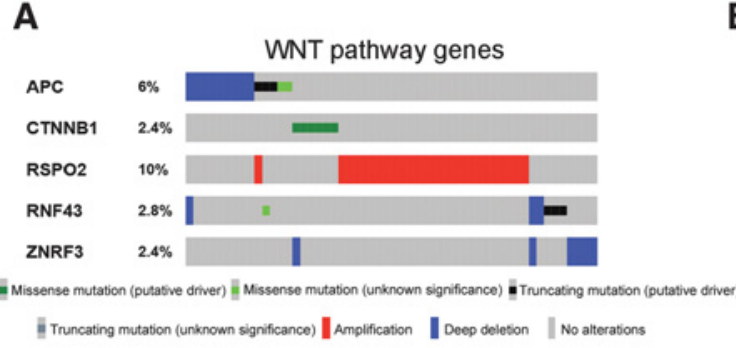

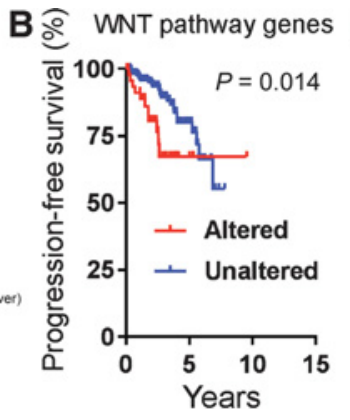
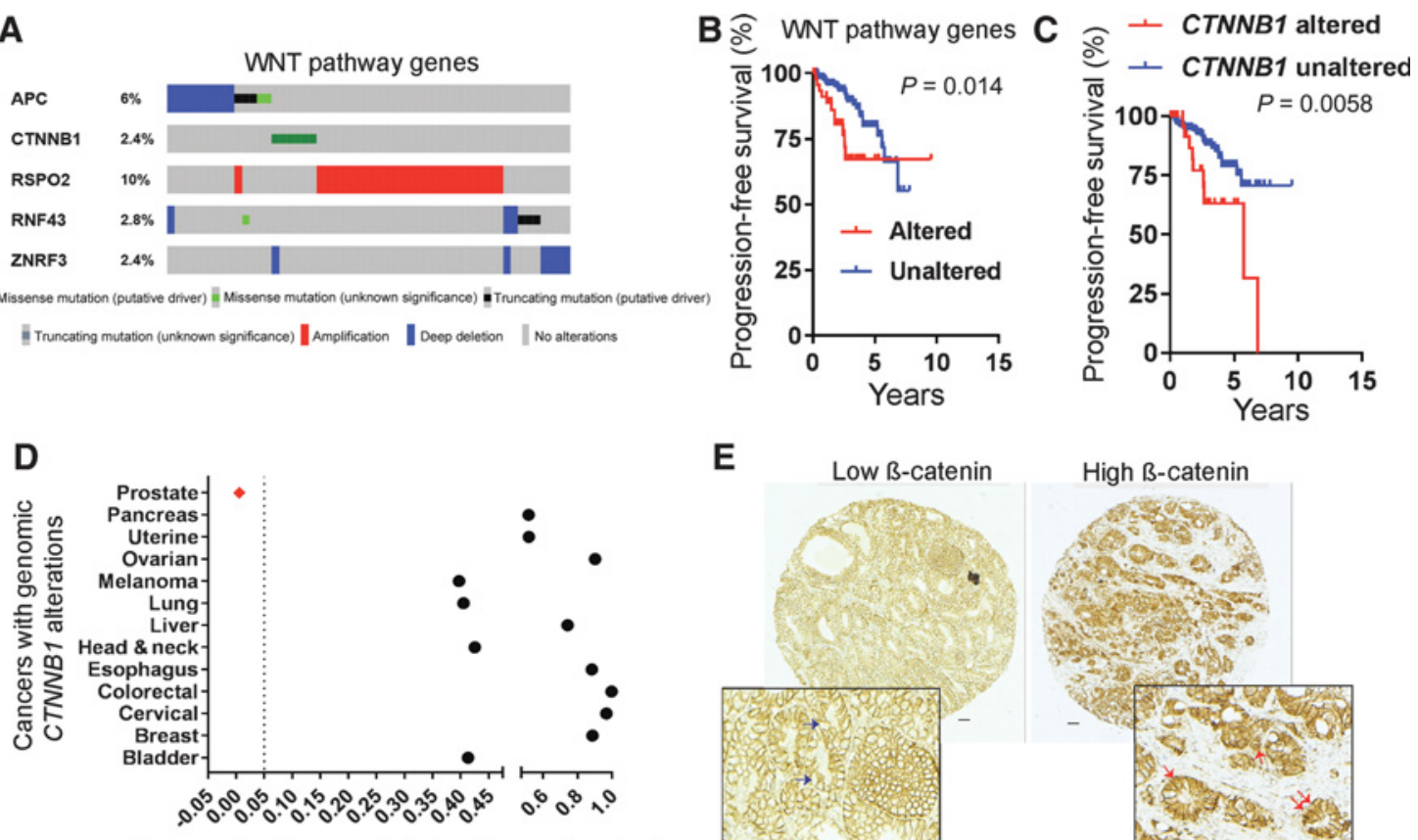

Progression-free survival significance ( $p$ value)
E Low B-catenin

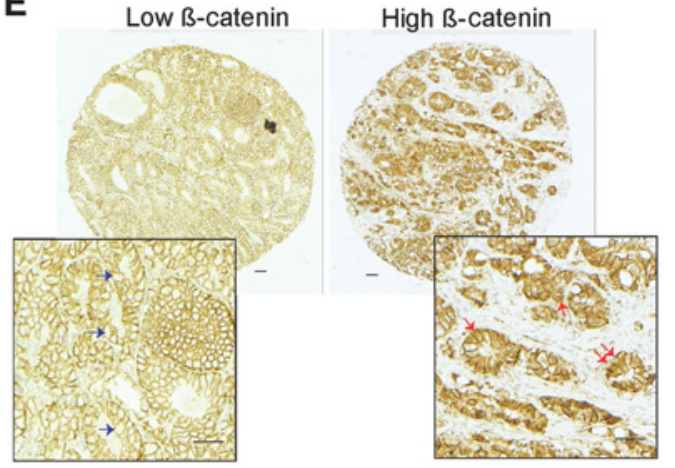

$\mathbf{F}$

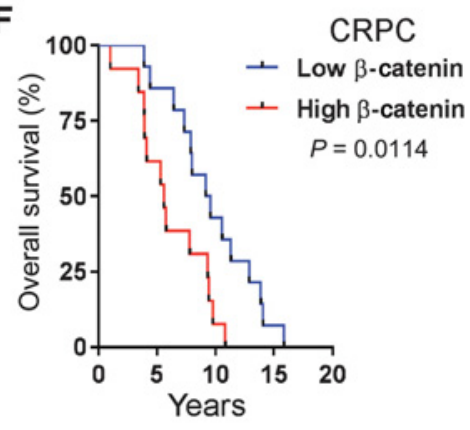

I

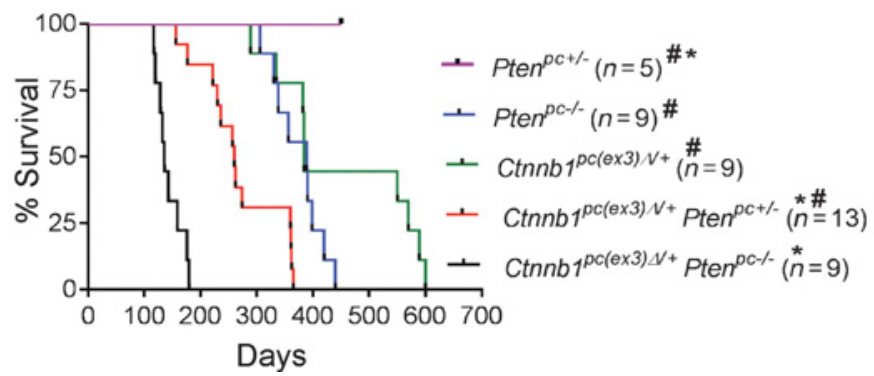

G $\quad r=-0.3555$

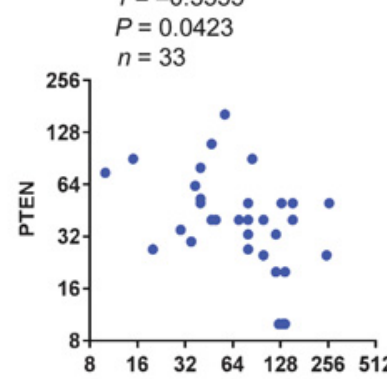

B-Catenin
H

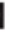
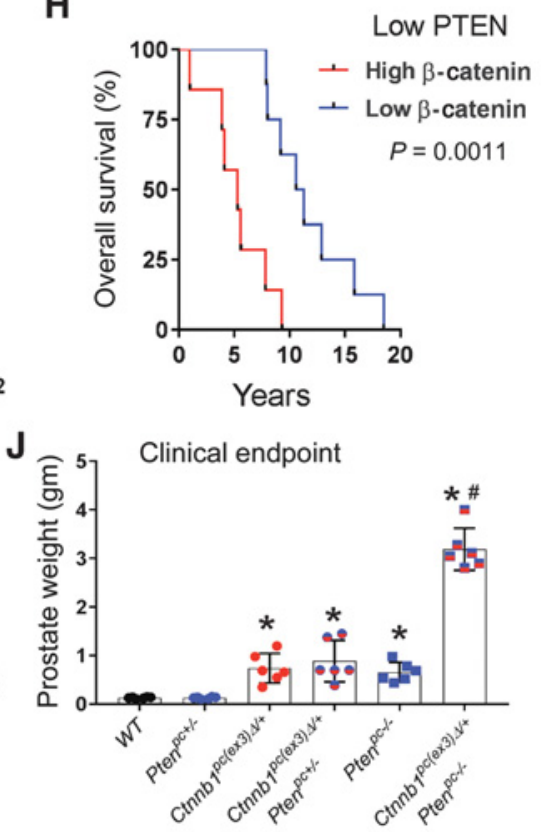

Figure 1.

Concurrent $B$-catenin activation and Pten loss in prostate cancer progression. A, Oncoprint showing canonical WNT pathway alterations in TCGA prostate cancer dataset $(n=251)$. B, Kaplan-Meier plot for progression-free survival in TCGA prostate cancer dataset showing cases with $(n=52)$ and without $(n=196)$ WNT pathway alterations. C, Kaplan-Meier plot for progression-free survival in TCGA prostate cancer dataset showing cases with ( $n=33$ ) and without ( $n=215)$ CTNNB1 (mutations, amplifications, and copy-number gain) alterations. D, The significance of CTNNB1 alterations by Kaplan-Meier analysis for the progression-free survival in the indicated cancer types. E, Representative IHC images of B-catenin in clinical prostate cancer TMA sections. Blue arrows, membrane staining without cytosolic or nuclear staining. Red arrows, membrane, cytosolic, and nuclear staining. F, Kaplan-Meier plot for post-diagnosis survival of patients with CRPC. B-Catenin-high (above median IHC score) patients, $n=13$; B-catenin-low (median and below median IHC score) patients, $n=14$. G, Scatter plot showing the correlation of $B$-catenin and PTEN IHC scores in the clinical prostate cancer samples. H, Kaplan-Meier plot for post-diagnosis survival of ADT-treated patients with low PTEN expression (below median IHC score) and high $\beta$-catenin (above median IHC score) patients, $n=7$, or low $\beta$-catenin (median and below median IHC score) staining, $n=8$. I, Kaplan-Meier plot for overall survival of mice with indicated prostate-specific genetic alterations driven by Probasin-Cre (pc). J, The prostate weight from indicated mice. ANOVA Tukey test; *, compared with $C \operatorname{tnn} b 7^{p c(e \times 3) \Delta /+}$;, compared with $C \operatorname{tnn} b 7^{p c(e \times 3) \Delta+}$ Pten $^{p c-/-}$. 
Patel et al.

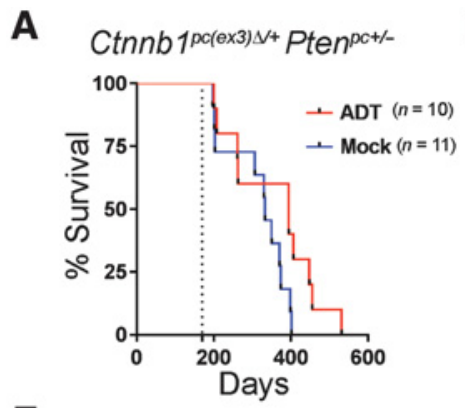

E
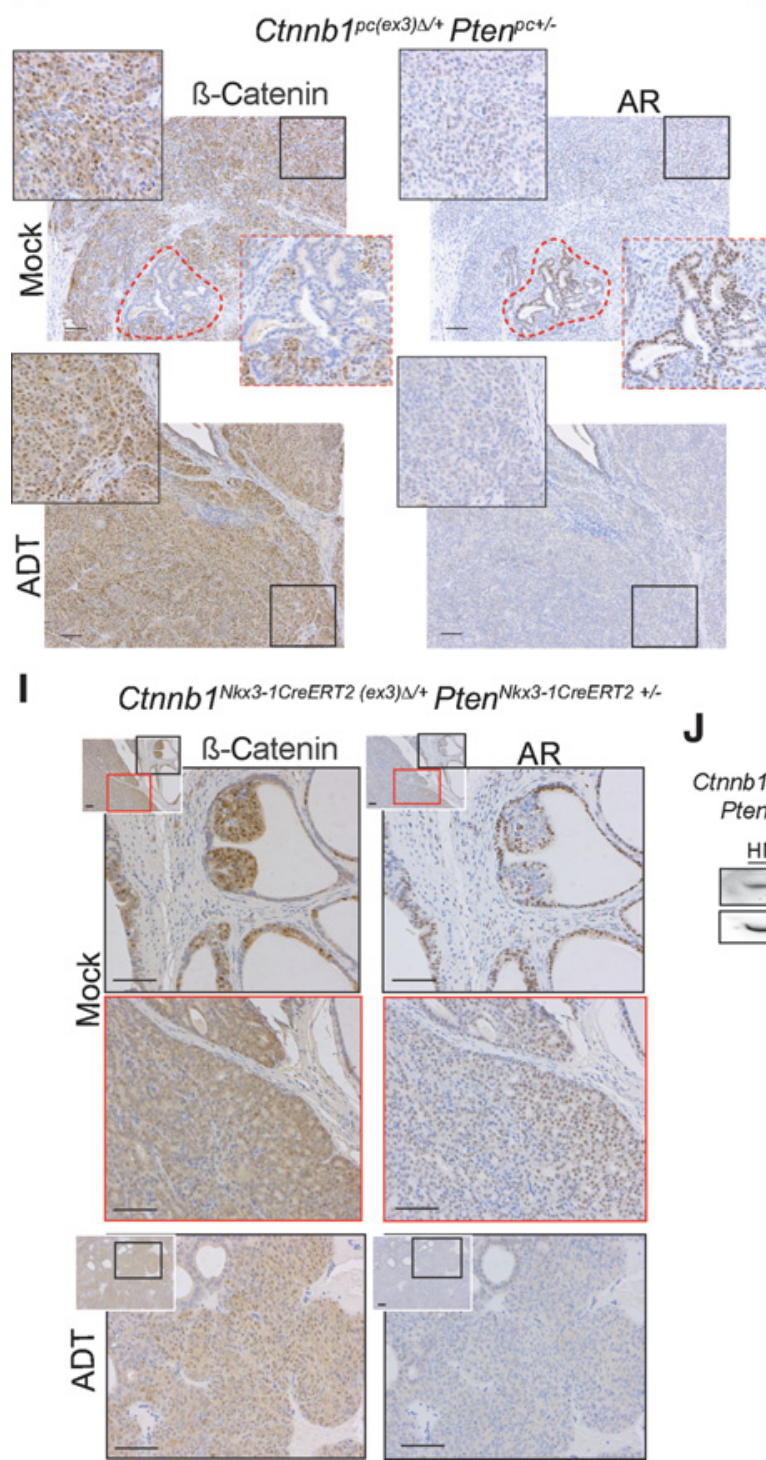
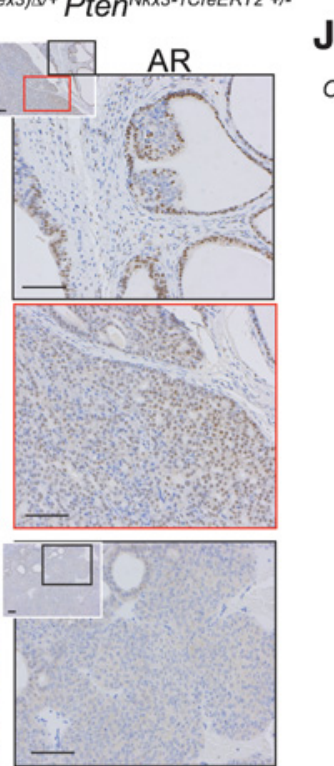

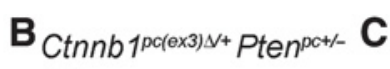

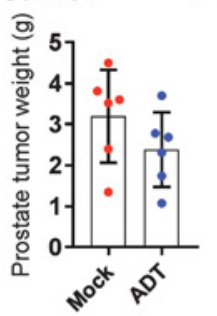

C Ctnnb 1 p( $($ (ex) 3$) \mathrm{N}+$

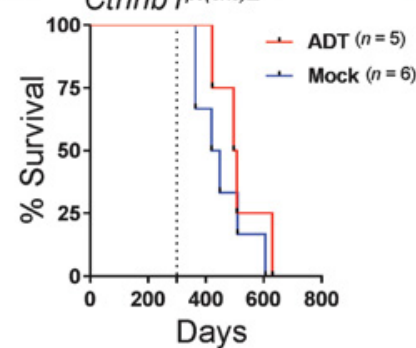

D Ctnnb $1^{10(-(x) \times)^{N+}}$
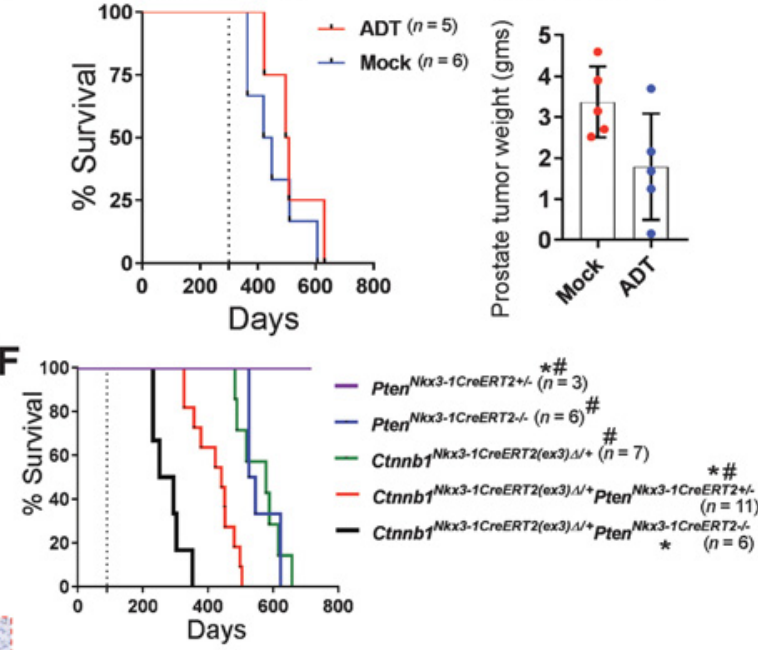

G

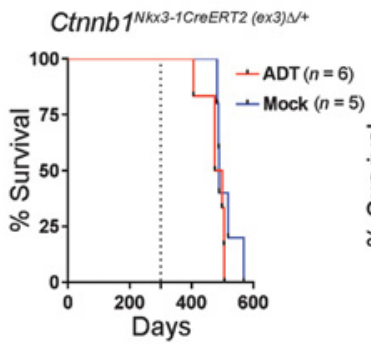

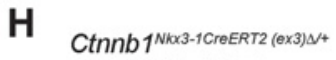

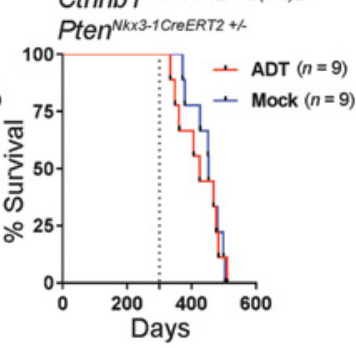

J Ctnnb1 $1^{\text {Nkx 3-1CreERT2 (ex3) N + }}$ Pten Nik3-1CreERT2 +\%

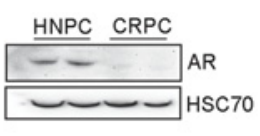

K

Ctnnb $1^{p c(e x 3) N_{+}}$ Pten ${ }^{p c+1-}$

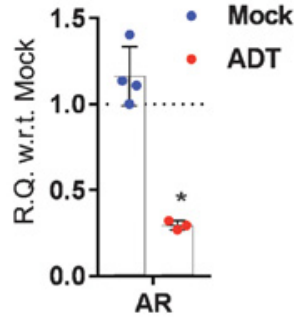

Figure 2.

B-Catenin activation drives AR-independent mCRPC. A, Kaplan-Meier plot for survival of Ctnnb $7^{\text {pc(ex3) } \Delta /+}{ }^{+}$ten ${ }^{p c+/-}$ mice treated with ADT when prostate tumor was palpable (150 days, dotted line). B, Prostate tumor weight at the clinical endpoint. C, Kaplan-Meier plot for survival of Ctnnb ${ }^{p c(e x 3) \Delta+}$ mice treated with ADT when the prostate tumor was palpable (300 days, dotted line). D, Prostate tumor weight at the clinical endpoint. E, Representative IHC images of prostate sections at clinical endpoint. F, Kaplan-Meier plot for survival of mice with indicated prostate-specific genetic alterations driven by tamoxifen-inducible NK 3 - 1 Cre

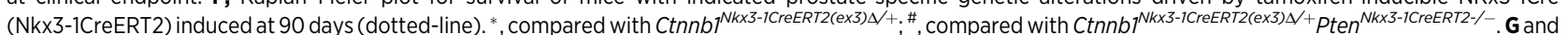

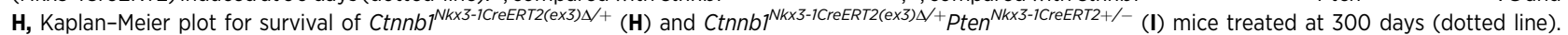
I, Representative IHC images of prostate sections. J, Representative immunoblots from hormone-naïve (HNPC) and castration-resistant (CRPC) prostate tumors. $\mathbf{K}$, AR mRNA quantification in prostate tumors (unpaired two-tailed Student $t$ test). 
homozygous (Pten ${ }^{\text {Nkx3-1CreERT2-/- }}$ Pten loss alone (Fig. 2F). Although Pten heterozygous mice developed PIN lesions at 1.5 years, the remaining genotypes developed AR-proficient tumors with varying latencies (Supplementary Fig. S3E). The survival and tumor burden were similar to that observed for the Probasin Cre-driven tumors (Fig 2F; Supplementary Fig. S3F). ADT treatment given at 300 days did not alter the survival in Ctnnb1 or double-mutant mice (Fig. 2G and $\mathbf{H}$ ). Highlighting the AR dependency in luminal cell-driven tumors, ADT significantly reduced prostate tumor burden in double-mutant mice (Supplementary Fig. S3G-S3I). Similar to Probasincre-driven tumors, at clinical endpoints, the $\mathrm{HN}$ prostate tumors from double-mutant Ctnnb1 ${ }^{\text {Nkx3-1CreERT2(ex3) } /+}{ }^{\text {Pten }}{ }^{\text {Nkx3-1CreERT2+/- }}$ mice had regions with varied AR proficiency, and the CRPCs had little detectable AR (Fig. 2I and J). Thus, independent of the cell of origin, Bcatenin activation and Pten haploinsufficiency led to AR-independent CRPC (Fig. 2J and K).

\section{B-Catenin activation antagonizes AR function}

The WNT/B-catenin and AR signaling cross-talk is unclear in prostate cancer. Hence, we studied the effect of mutant $B$-catenin on AR function by investigating the expression of AR target genes in Ctnnb1 $^{\text {Nkx3-1CreERT2(ex3) } \Delta /+}$ Pten $^{\text {Nkx3-1CreERT2+/- }}$ tumors (27). Compared with WT prostates, the AR target gene expression was lower in $\mathrm{HN}$ double-mutant tumors and was comparable between $\mathrm{HN}$ and CRPC double-mutant tumors (Fig. 3A). Confirming lower AR function, the AR occupancy on the promoter regions of AR target genes and the total AR peaks were reduced in tumors with $B$-catenin activation (Fig. 3B-D; Supplementary Fig. S3J). In the double-mutant tumors, truncated active $\beta$-catenin binds to AR (Fig. 3E). Compared with WT prostates, both Fkbp5 expression and AR binding to the promoter of Fkbp5, an AR target gene, were significantly decreased in doublemutant prostate tumors (Fig. 3F). Collectively, our data indicate loss of AR function in Ctnnb1-mutant tumors with Pten haploinsufficiency.

We castrated mice 30 days after induction to investigate if activated B-catenin can mediate AR-independent prostate tumorigenesis by bypassing the AR pathway (Fig. 3G). At 300 days, WT and Pten heterozygous castrated prostates showed complete regression with no nuclear B-catenin (Fig. 3H). The Ctnnb1-mutant prostates from Ctnnb1 $1^{\text {Nkx3-ICreERT2(ex3) } \Delta++}$ mice also showed complete regression with only a few prominent nuclear B-catenin-mutant cells (black arrows, Fig. $3 \mathbf{H})$. Highlighting the dispensability of AR signaling in Pten-null prostates, three out of four castrated prostates showed AR-deficient PIN lesions with membranous B-catenin staining (Fig. 3H; Supplementary Fig. S3K). Prostates from castrated Ctnnb1 $^{\text {Nkx3-1CreERT2(ex3) } /+}$ Pten $^{\text {Nkx3-1CreERT2+/- }}$ mice showed PIN lesions with prominent nuclear $B$-catenin (Fig. 3H; Supplementary Fig. S3K). Emphasizing the cooperation between Ctnnb1-activating mutation and Pten deletion in prostate tumor progression, a majority of castrated Ctnnb $1^{\text {Nkx3-1CreERT2(ex3) } /+}{ }^{\text {Pten }}{ }^{\text {Nkx3-1CreERT2-I- }}$ mice developed poorly differentiated AR-deficient tumors (Fig. 3H; Supplementary Fig. S3K). Thus, independent of the cell of origin, B-catenin activation cooperates with Pten loss to drive AR pathwayindependent prostate cancer.

We next investigated the expression of $B$-catenin target genes in double-mutant prostate tumors. Compared with Pten-null prostate tumors, the double-mutant tumors, where tumoral Pten levels were similar to Pten-null tumors (Supplementary Fig. S1G and S1H), showed a significant increase in expression of $ß$-catenin target genes such as c-Myc, Tiam1, Axin2, and Dkk1 (Fig. 3I), with no significant differences between $B$-catenin-mutant tumors with heterozygous or homozygous Pten loss (Supplementary Fig. S3L). Although c-Myc and
Axin2 expression was sustained in double-mutant CRPC following $\mathrm{ADT}$, expression of other $B$-catenin target genes was significantly altered compared with $\mathrm{HN}$ tumors (Fig. 3J). These data may suggest a potential mutual antagonism between $ß$-catenin and AR pathways.

\section{WNT signaling is enriched in Ctnnb1-mutant prostate tumor models}

To investigate the growth advantages conferred by mutant Ctnnb1 in prostate cancer, we established spheroid models from Pten-null

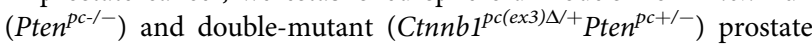
tumors, with two cell lines from each genotype referred to as P1, P2 and $\mathrm{CP} 1, \mathrm{CP} 2$ respectively (Fig. $\mathbf{4 A - C}$ ). Overall, CP1 and CP2 formed invasive and nonpolarized spheroids compared with the smaller and well-polarized P1 and P2 spheroids (Fig. 4B and C). Although the P1 spheroids showed membranous $B$-catenin, the $\mathrm{CP}$ spheroids showed invasive spheroids with prominent nuclear $B$-catenin (Fig. 4B and C). Both $\mathrm{P}$ and $\mathrm{CP}$ spheroids were Pten deficient, and $\mathrm{CP}$ spheroids showed truncated B-catenin (Fig. 4D; Supplementary Fig. S4A). The epithelial Pten DNA levels were significantly lower in double-mutant $\left(C t n n b 1^{p c(e x 3) \Delta /+}\right.$ Pten $\left.^{p c+/-}\right)$ tumors compared with Pten heterozygous $\left(\right.$ Pten $\left.^{p c+/-}\right)$ prostates, suggesting a possible further genomic loss of Pten in double-mutant tumors (Supplementary Fig. S4B).

Compared with $\mathrm{P}$ cells, CP cells formed significantly bigger prostate tumors with increased visceral metastases and shorter survival in immunodeficient mice (Fig. 4E and F; Supplementary Fig. S4C$\mathrm{S} 4 \mathrm{H})$. The prostate tumors from $\mathrm{P}$ cells were cystic with membranous $\beta$-catenin, and $\mathrm{CP}$ prostate tumors were poorly differentiated with prominent nuclear $\beta$-catenin staining (Fig. 4F).

We next compared the transcriptomic profiles of Pten-null $\left(\right.$ Pten $\left.^{p c-1-}\right)$ and Ctnnb1-mutant tumors $\left(C_{t n n b} 1^{p c(e x 3) \Delta /+}\right.$ and $C t n n b 1^{p c(e \times 3) \Delta /+} P_{t e n}{ }^{p c+/-}$ ) with WT prostates to identify genes upregulated only in tumors with $\beta$-catenin activation (Fig. 4G). B-Cateninupregulated clusters were enriched for WNT and metabolic signaling (Supplementary Fig. S4I). Transcriptomic comparison of P and CP tumors showed enrichment of WNT signaling in ß-cateninupregulated cluster enriched with Wnt5a as one of the enriched upstream regulators (Fig. 4G; Supplementary Fig. S4J and S4K). R-spondin2 (RPOS2) amplifications occur in about half of the prostate cancer cases with WNT/ß-catenin pathway gene alterations (Fig. 1A). RPOS2 potentiates WNT signaling by binding to the LGR receptors. Although RPOS2 expression remains unaltered, the expression of LGR5 and 6 is significantly higher in Ctnnb1-mutant prostate tumors compared with Pten-null tumors (Fig. 4H). Suggestive of an increased WNT signaling, the CRPC from ADT-treated Ctnnb $1^{p(\operatorname{ex} 3) \Delta /+} P \operatorname{ten}^{p c \pm}$ has increased expression of RSPO2 and LGR5 and 6 compared with mock tumors (Fig. 4I).

Because WNT signaling was enriched, we examined the expression of WNT ligands in the Ctnnb1-mutant models. The transcriptomic profiles of WNT genes in prostate tumors, metastases, and CRPC showed enrichment of a subset of WNT ligands in ß-catenin-mutant tumors (Fig. 4J; Supplementary Fig. S4L-S4N). Among the WNTs examined, only WNT5A enrichment significantly associated with poor prognosis and positively correlated with CTNNB1 expression (Fig. 4K and L; Supplementary Fig. S4O).

\section{Wnt5a sustains the growth of Ctnnb1-mutant prostate cancer cells}

We find WNT5a, a noncanonical WNT ligand, to be clinically relevant and enriched in prostate tumors with $\beta$-catenin activation. Hence, we investigated the functional relevance of Wnt5a in tumors with $ß$-catenin activation. The Wnt5a expression was significantly 


\section{Patel et al.}
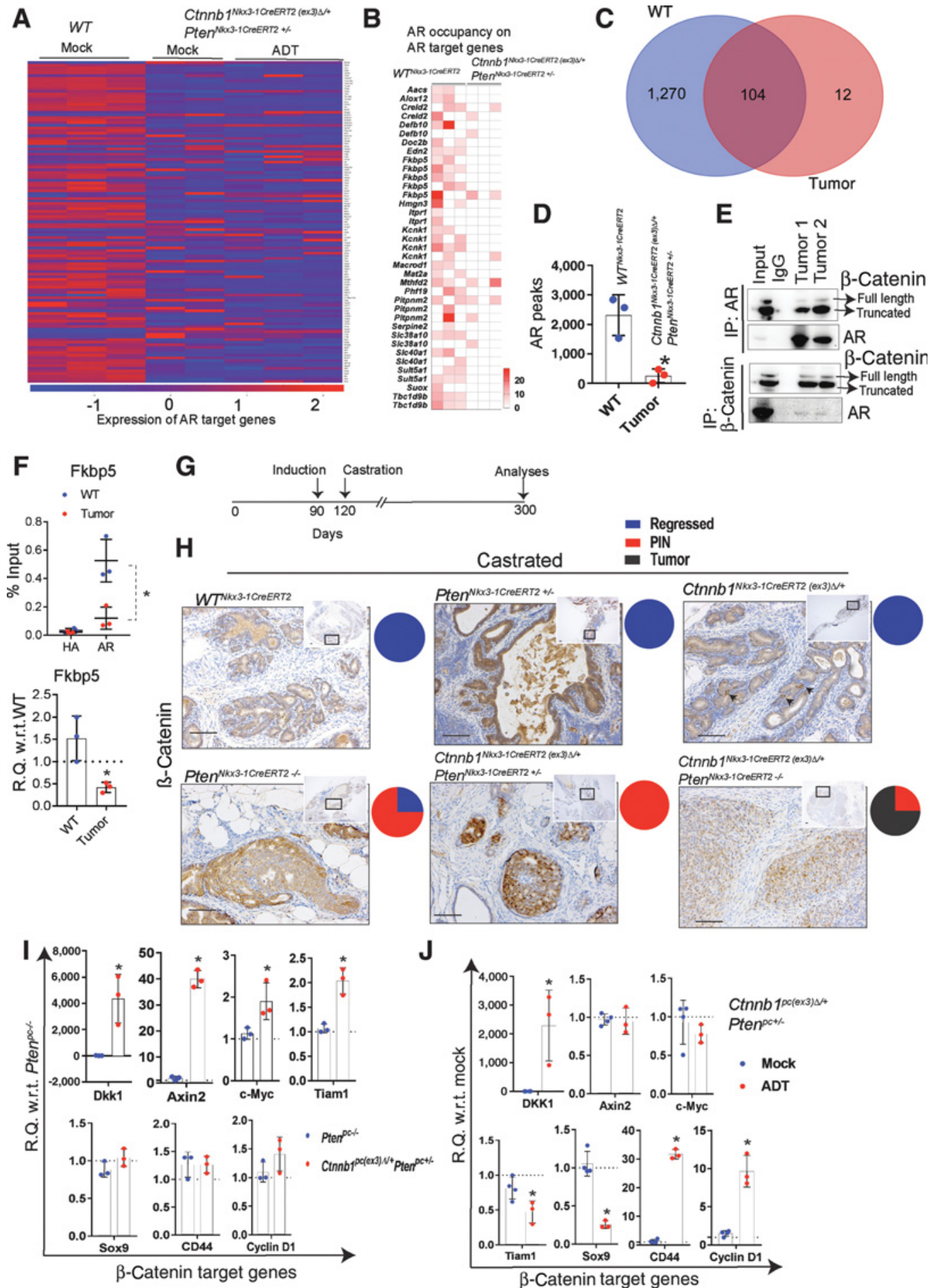

Figure 3.

Mutant B-catenin antagonizes AR function. A, Heat map showing AR target genes' expression in WT prostates and prostate tumors. B, Heat map showing AR enrichment on promoters of AR target genes in 10-month-old prostates and tumors (scale, fold enrichment relative to input). C, Venn diagram showing AR-bound genes in WT prostates and Ctnnb1 $1^{N k \times 3-1 C r e E R T 2(e \times 3) \Delta+}{ }^{+}$ten ${ }^{N k \times 3-1 C r e E R T 2+/-}$ tumors. D, Total AR-bound peaks relative to input. E, Representative immunoprecipitation immunoblots from Ctnnb7 $7^{N k \times 3-1 C r e E R T 2(e \times 3) \Delta+}{ }^{2}$ ten $^{N k \times 3-1 C r e E R T 2+/-}$ tumor lysates. F, Top, enrichment of AR on Fkbp5 promoter in WT $T^{N k \times 3.1 C r e E R T 2}$ and

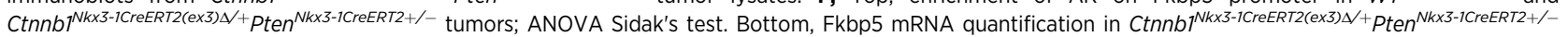
tumors relative to $W T^{N k \times 3.1 C r e E R T 2}$. G, Schematic of early castration experiment where mice were castrated 30 days after induction and prostate tissues were analyzed at day 300. H, Representative IHC images of prostate sections from ADT-treated mice as indicated. ADT consequences on prostates $(n=4)$ represented as pie charts. I-J, mRNA quantification of B-catenin target genes. Unpaired two-tailed Student $t$ test for $\mathbf{D}, \mathbf{F}, \mathbf{I}$, and $\mathbf{J} .{ }^{*}, P<0.05$. 
A

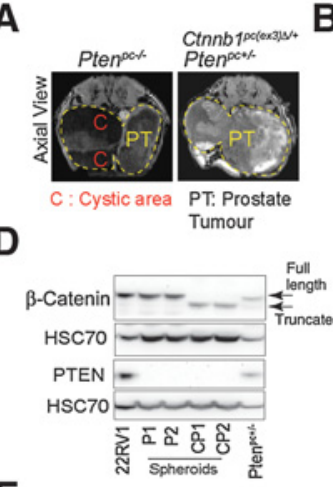

E

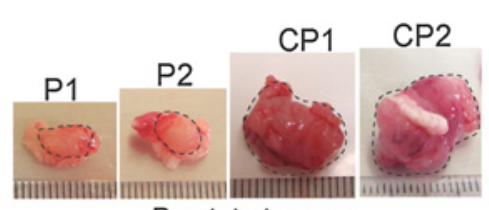

Prostate tumors

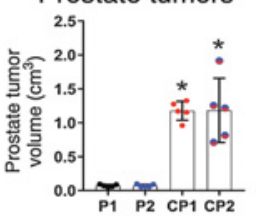

G
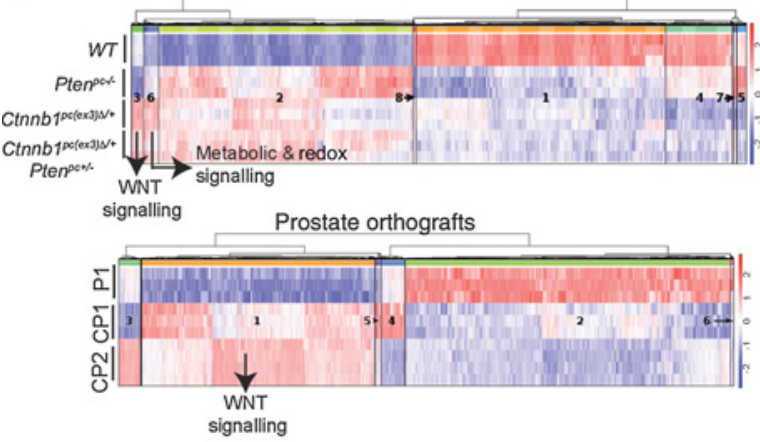

J

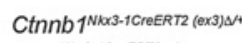

Pten ${ }^{\text {Nkx3-1CreERT2 +\% }}$ HNPC CRPC

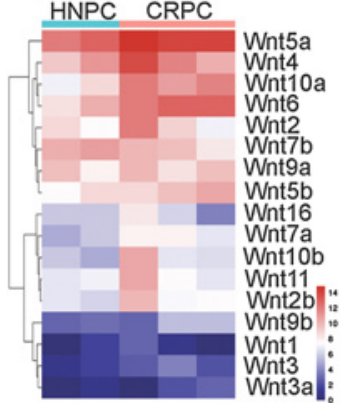

$\mathbf{F}$
K

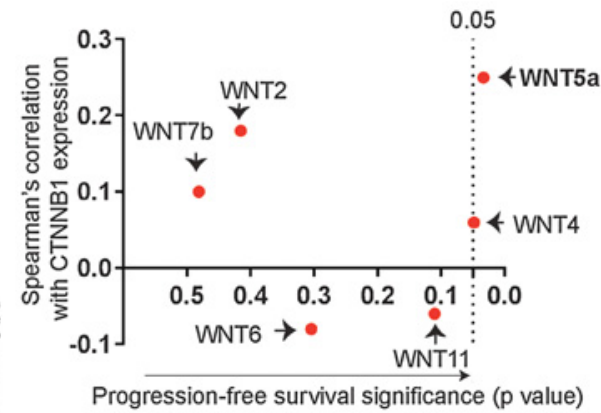

C

C Phalloidin +
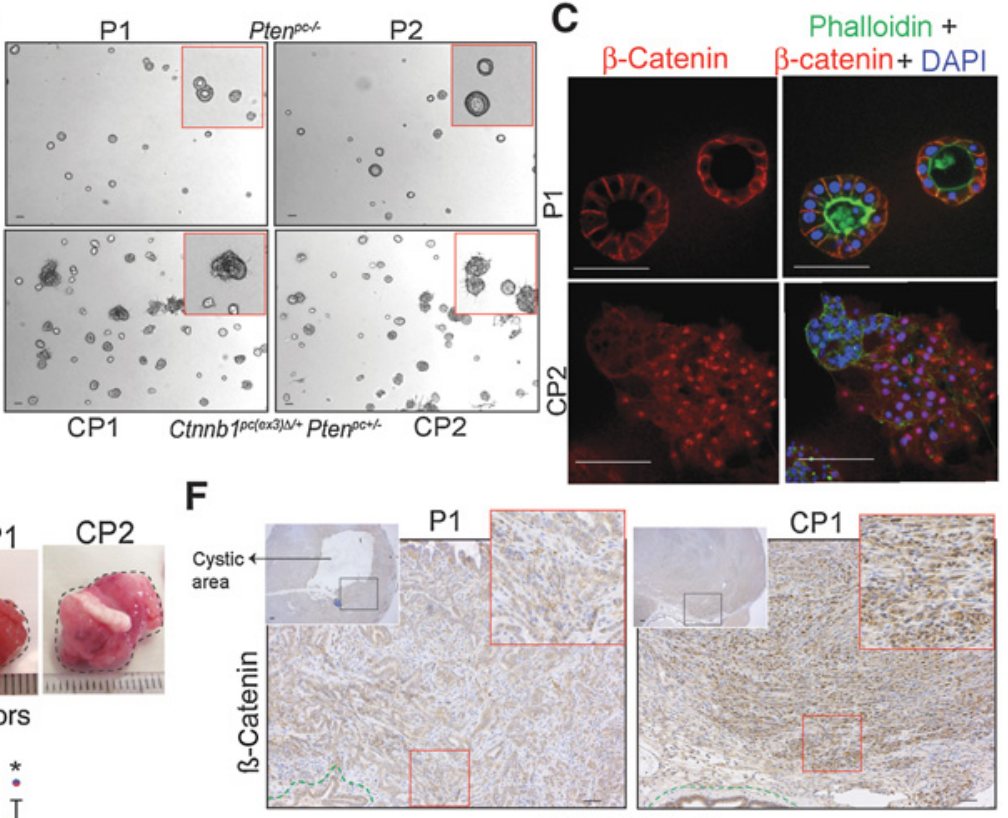

Prostate tumors

H

I

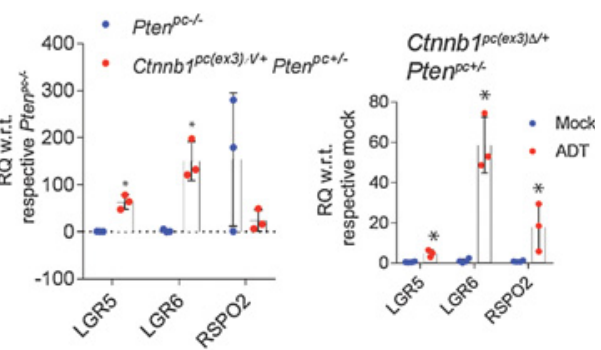

$\mathbf{L}$

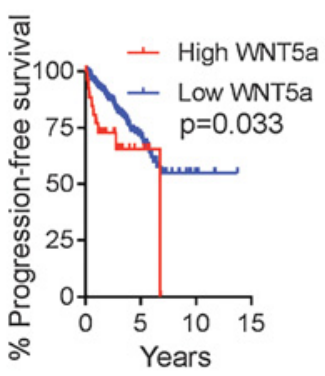

Figure 4.

Ctnnb1-mutant prostate tumor models are enriched for WNT signaling. A, Magnetic resonance imaging axial view of prostate tumors. B, Prostate tumor spheroid cultures established from two independent Pten $^{p c-/-}(\mathrm{P} 1, \mathrm{P} 2)$ and Ctnnb $7^{p c(e \times 3) \Delta+}$ Pten $^{p c+/-}(\mathrm{CP1}, \mathrm{CP} 2)$ mice. C, Representative immunofluorescence cross-section of $\mathrm{P} 1$ and CP2 spheroid. D, Representative immunoblot as indicated (HSC70 was loading control). E, Representative prostate tumor photos (top plot) and prostate tumor volumes (bottom plot) generated using P1, P2, CP1, and CP2 cells. ANOVA Tukey test. Black dotted areas, prostate orthograft tumor. F, Representative IHC images of prostate tumors. G, Transcriptomic clustering heat map of WT prostate and tumors. H and I, mRNA quantification in prostate tumors. J, Heat map of log $2^{-}$ transformed and DESeq2-normalized count matrices from RNA-seq. K, Dot plot showing the effects of WNT enrichment ( $z$-score $>1.4)$ on progression-free survival and correlation of WNT and CTNNB1 expression in TCGA prostate cancer dataset. L, Kaplan-Meier plot for progression-free survival in TCGA prostate cancer dataset, with high $(n=28)$ or low $(n=464)$ WNT5a expression. Unpaired two-tailed Student $t$ test for $\mathbf{H}$ to $\mathbf{I}$. ${ }^{*}, P<0.05$. 
Patel et al.

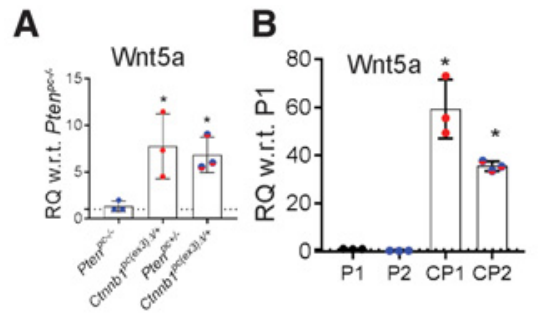

E

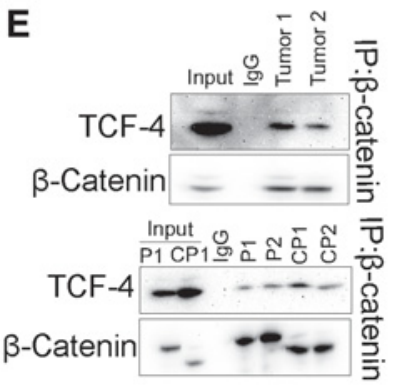

H

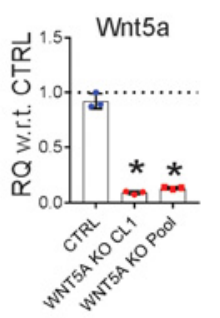

I $\rightarrow$ CTRL - WNT5AKO CL1 - - WNT5AKO POOI

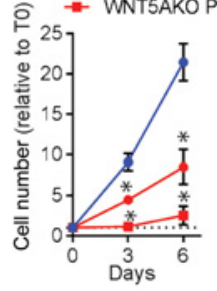

F

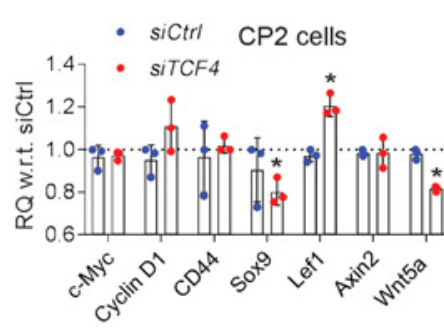

D $\quad$ CP2 cells

- sictrl

$\left.E^{2.0}\right] \cdot$ sictnnb 1

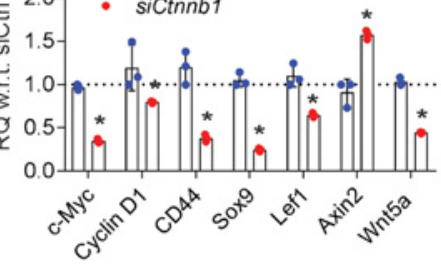

G

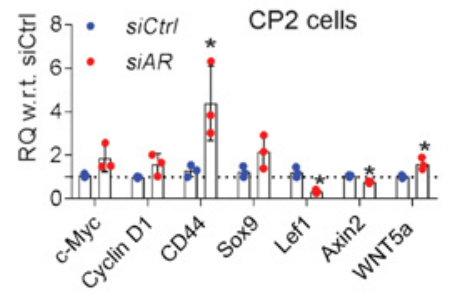

L CP2 cells

- cTRL

- Wnt5a ko Cl1
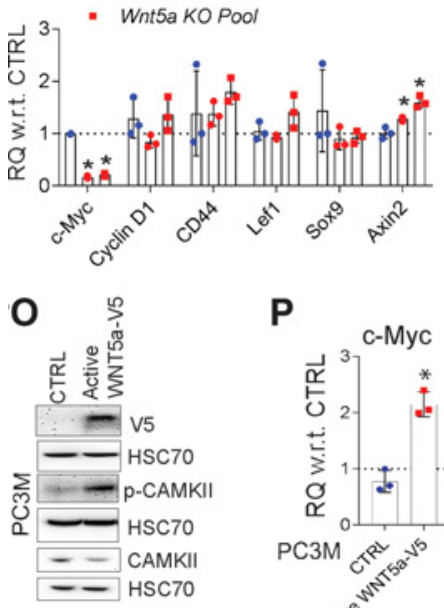

J

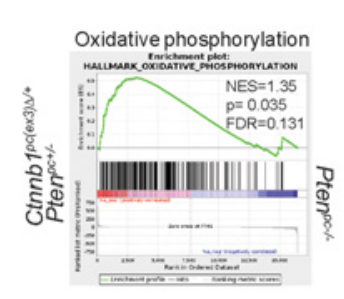

K

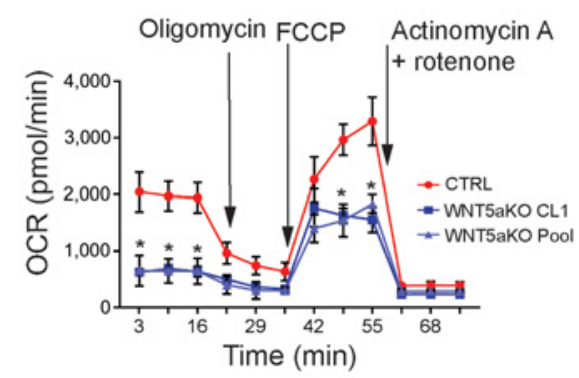

M

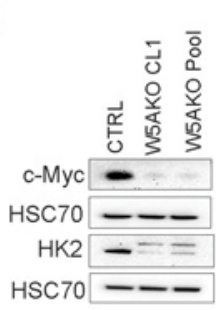

N
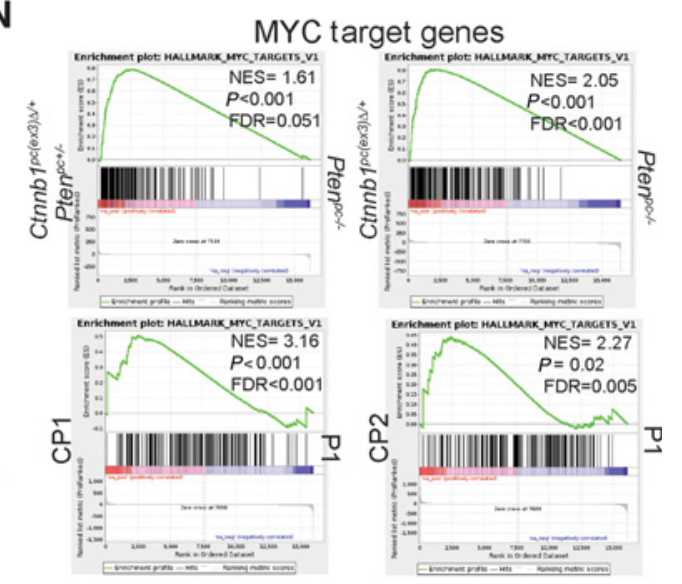

Figure 5.

WNT5a sustains the growth of Ctnnb1-mutant prostate cancer cells. A and B, WNT5a mRNA quantitation in prostate tumors (A) and orthograft (B). ANOVA Dunnett's (A) and Tukey (B) tests. C, WNT5a mRNA quantitation in prostate tumors. D, mRNA quantification in CP2 cells with B-catenin knockdown. E, Representative immunoprecipitation immunoblots from $C t n n b 7^{p c(e \times 3) \Delta /+} P t e n^{p c+/-}$ tumor lysates (top) and murine prostate cancer cell lines (bottom). $\mathbf{F}$ and $\mathbf{G}$, mRNA quantification in CP2 cells with TCF-4 (F) or AR (G) knockdown. H, WNT5a mRNA quantitation in WNT5a KO CP2 cells. I, Growth rate of WNT5a KO CP2 cells. J, GSEA in prostate tumors. K, OCR in WNT5a KO cells. $\mathbf{L}$ and $\mathbf{M}$, mRNA quantitation (L) and immunoblots (M) in WNT5a KO cells. N, GSEA of MYC targets in prostate tumors. $\mathbf{O}-\mathbf{Q}$, Representative immunoblots ( $\mathbf{O})$, c-Myc mRNA quantification (P), and growth rate $(\mathbf{Q})$ in PC3M cells with active WNT5a expression. Unpaired two-tailed Student $t$ test for $\mathbf{C}, \mathbf{D}, \mathbf{F}, \mathbf{G}$, and $\mathbf{P}$ and ANOVA Tukey test for $\mathbf{H}, \mathbf{I}, \mathbf{K}, \mathbf{L}$, and $\mathbf{Q}$. ${ }^{*}, P<0.05$. 
elevated in Ctnnb1-mutant prostate tumors compared with Pten-null tumors (Fig. 5A and B). Importantly, CRPC tumors from both Probasin- and Nkx3-1 Cre-driven double-mutant mice showed a significant increase in Wnt5a expression when compared with $\mathrm{HN}$ tumors (Fig. 5C). Wnt5a RNA scope (RNA-ISH) confirmed the enhanced Wnt5a expression in the Ctnnb1-mutant CRPC tumor epithelium (Supplementary Fig. S5A). Similar to $B$-catenin target genes, $B$-catenin knockdown significantly decreased Wnt5a expression in CP2 cells (Fig. 5D; Supplementary Fig. S5B). Among B-cateninbinding partners, TCF4 binds to B-catenin in the Ctnnb1-mutant models (Fig. 5E). Similar to ß-catenin knockdown, TCF4 knockdown also significantly decreased Wnt5a expression (Fig. 5F; Supplementary Fig. S5C). Other TCF family members, TCF7 and TCF7L1, did not significantly alter the expression of $B$-catenin target genes (Supplementary Fig. S5D). Thus, activated $B$-catenin may bind to TCF4 to induce Wnt5a expression. Interestingly, AR knockdown significantly increased Wnt5a expression in CP2 cells (Fig. 5G; Supplementary Fig. S5E). Thus, Wnt5a expression may be induced by $ß$-catenin activation and inhibited by AR.

To further investigate the functional contribution of Wnt5a, we generated Wnt5a CRISPR knockout (KO) CP2 cells (Fig. 5H). Compared with CP2 control cells, Wnt5a KO CP2 cells showed impaired proliferation (Fig. 5I). GSEA showed increased enrichment of oxidative phosphorylation in Ctnnb1-mutant prostate tumors with Pten loss compared with Pten-null tumors (Fig. 5J). The metabolic analyses of spent-media from CP1 and CP2 Ctnnb1-mutant cells showed increased glucose consumption, and a stable isotope tracing experiment showed enriched ${ }^{13} \mathrm{C}_{6}$-glucose derived metabolites in Ctnnb1mutant CP2 cells (Supplementary Fig. S5F-S5H). Overall, compared with Pten-null cells, the Ctnnb1-mutant cells had elevated glucose catabolism, whereas Wnt5a KO significantly decreased OCR and ECAR, indicating reduced oxidative phosphorylation and glycolysis (Fig. 5K; Supplementary Fig. S5I).

Because Wnt5a can both activate and repress Wnt/ $\beta$-catenin signaling (28), we next studied the expression of $ß$-catenin target genes in Wnt5a KO cells (Fig. 5L). Among ß-catenin target genes, Wnt5a KO only decreased the expression of c-Myc (Fig. 5L). Furthermore, Wnt5a loss decreased the expression of hexokinase 2 (HK2), an Myc transcriptional target, that catalyzes the rate-limiting step of glucose metabolism (Fig. 5M). Importantly, both Ctnnb1-mutant and double-mutant tumors (and CP orthografts) show enrichment for MYC target genes compared with Pten-null tumors (Fig. 5N). Thus, c-Myc may act a mediator of $\mathrm{WNT} / \beta$-catenin signaling in prostate cancer (29). Because c-Myc is a critical regulator of cellular metabolism (30), by sustaining Myc expression, Wnt5a may support the metabolism and growth of Ctnnb1-mutant prostate cancer cells. Active WNT5a expression in human PC3M prostate cancer cells [as shown by increased phosphorylation of $\mathrm{Ca}^{2+} /$ calmodulin-dependent protein kinase II (CAMKII) - an effector of WNT5a-induced signaling] increased c-Myc expression and growth rate compared with control cells (Fig. 5O-Q).

Thus, Wnt5a is induced by activated $B$-catenin and may play a functional role in sustaining the growth of Ctnnb1-mutant cells.

\section{WNT5a sustains c-Myc and inhibits AR expression}

We show that $B$-catenin regulates c-Myc expression in Ctnnb1mutant cells (Fig. 5D). Hence, we studied the cellular distribution of $ß$-catenin in Wnt5a KO cells. WNT5a KO decreased the nuclear levels of $B$-catenin and increased AR expression in CP2 cells (Fig. 6A and B). Because NFKB regulates AR expression and WNT5a induces NFKB activation (31), we investigated cellular distribution of NFKBp65
(RelA) in Wnt5a KO cells. WNT5a loss increased cytosolic and decreased nuclear levels of NFKBp65 (RelA; Fig. 6C). Confirming the role of $B$-catenin and NFKBp65 in AR repression, $B$-catenin or NFאBp65 knockdown increased AR expression in CP2 cells (Fig. 6D). These data suggest that by regulating nuclear localization of $B$-catenin, WNT5a sustains c-Myc expression and also induces nuclear NFאBp65 localization to inhibit AR expression. WNT5a loss also decreased the expression of LGR5 and 6 in Ctnnb1-mutant prostate cancer cells (Fig. 6E). Importantly, AR knockdown increased the expression of LGR5 and 6 (mediators of WNT signaling) in WNT5a KO cells (Fig. 6F). Together, our data suggest that $ß$-catenin-induced WNT5a enhances the expression of LGR5 and 6 by repressing AR expression.

We next investigated the WNT/B-catenin and AR mutual antagonism in Ctnnb1-mutant murine prostate cancer spheroid and orthograft models. Although the Ctnnb1-mutant spheroids were resistant to enzalutamide and WNT5a loss decreased their growth, WNT5a loss combined with enzalutamide further decreased spheroid formation (Supplementary Fig. S6A). Although the survival was higher in mice bearing WNT5a KO tumors, Wnt5a KO CP tumors at endpoint were significantly smaller only in ADT-treated mice (Fig. 6G and $\mathbf{H}$; Supplementary Fig. S6B-S6D). Thus, the combined loss of WNT5a and ADT inhibited the growth of Ctnnb1-mutant tumors. Next, we investigated the impact of WNT5a on regulating AR function. Active WNT5a expression in human C4-2 CRPC cells decreased the levels of AR targets such as PSA and FKBP5 (Fig. 6I). As a consequence of decreased AR function, C4-2 cells proliferated at a lower rate in the presence of active WNT5a (Fig. 6I and J). Highlighting the AR dependency of C4-2 CRPC cells, AR knockdown reduced the growth of C4-2 cells (Fig. 6J). In contrast, compared with control cells, the active WNT5a-expressing C4-2 cells showed sustained proliferation in the absence of AR (Fig. 6J). Interestingly, ARdeficient WNT5a-expressing C4-2 cells showed increased levels of p-CAMKII, an effector of noncanonical WNT5a function (Fig. 6I). Thus, AR may inhibit the noncanonical function of WNT5a, such as $\mathrm{Ca}^{2+} /$ CAMKII signaling. Overall, AR and WNT5a exhibit mutual antagonism.

We show that WNT5a-mediated nuclear localization of $ß$-catenin and NFKBp65 sustains c-Myc and represses AR expression, respectively. Our data indicate a reciprocal inhibition between WNT/ßcatenin and AR signaling and highlight the importance of dual targeting of both pathways in prostate cancer with $ß$-catenin activation (Fig. 6K).

\section{Dual targeting of AR and WNT signaling in Ctnnb1-mutant prostate cancer}

To confirm the mutual antagonism between $\mathrm{AR}$ and $\mathrm{WNT} / \mathrm{B}$ catenin signaling, we targeted these pathways in luminal-derived

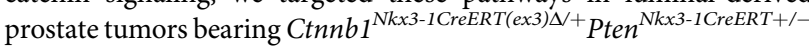
mice. We have shown that Ctnnb1-mutant tumors depend on WNT signaling for sustained growth and hypothesize that inhibition of WNT secretion using the PORCN inhibitor LGK974 would diminish the growth advantage conferred by WNT ligands including WNT5a. Therefore, we treated the Ctnnb1 $1^{\text {Nk } k \text { - ICreERT(ex) } 3 / 4+}$ Pten ${ }^{\text {Nkx3-1CreERT+1- }}$ double-mutant mice with ADT followed by LGK974 to inhibit WNT secretion (Supplementary Fig. S7A). Compared with ADT alone, which resulted in ARindependent CRPC, the combined ADT and LGK974 treatment decreased tumor burden and proliferation, with residual tumor epithelium showing high levels of nuclear B-catenin (Fig. 7A and B; Supplementary Fig. S7B). Thus, LGK974 treatment may restrict the proliferation of ADT-treated Ctnnb1-mutant tumors by limiting WNT 
Patel et al.
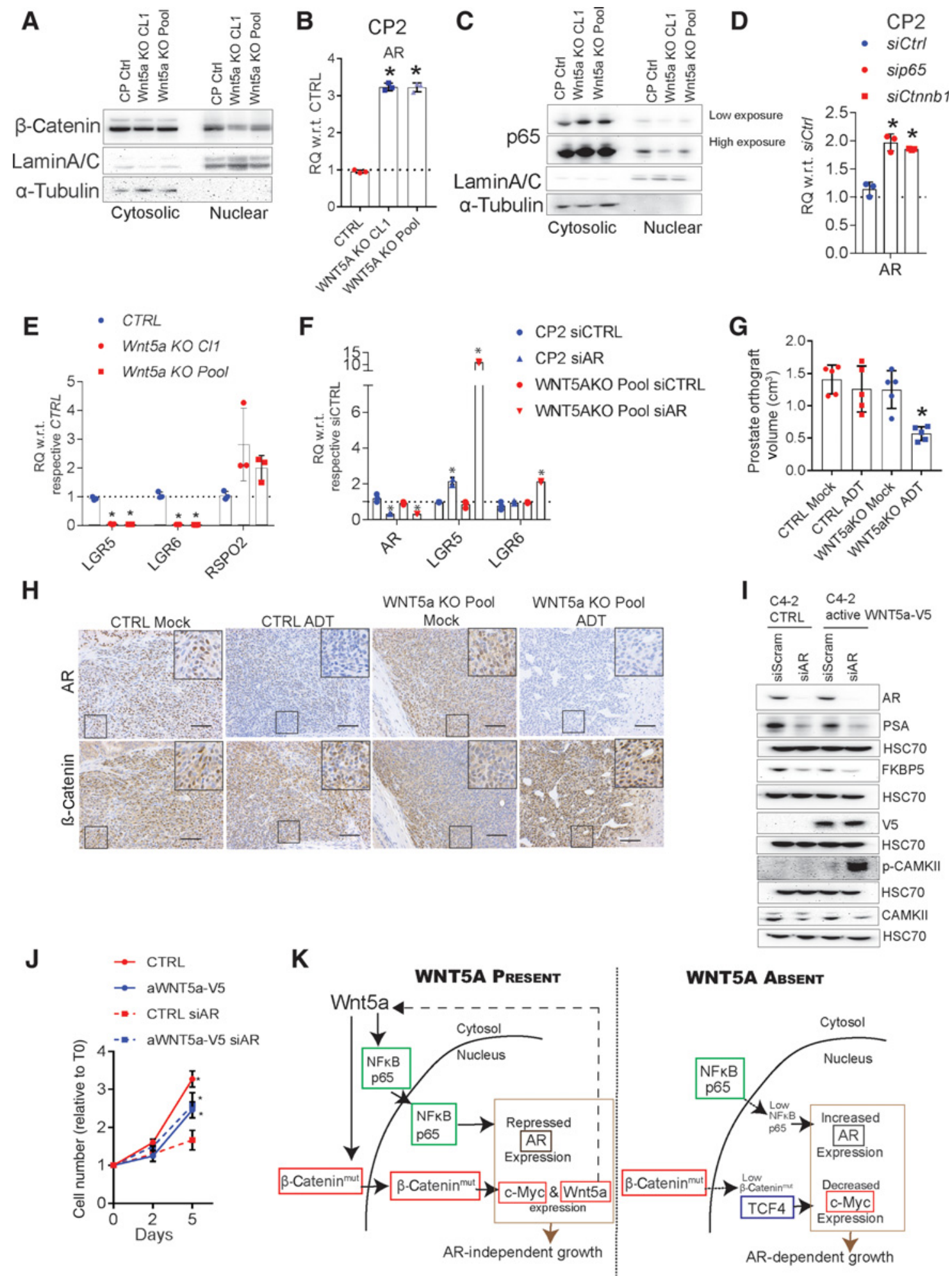

WNT5A Absent

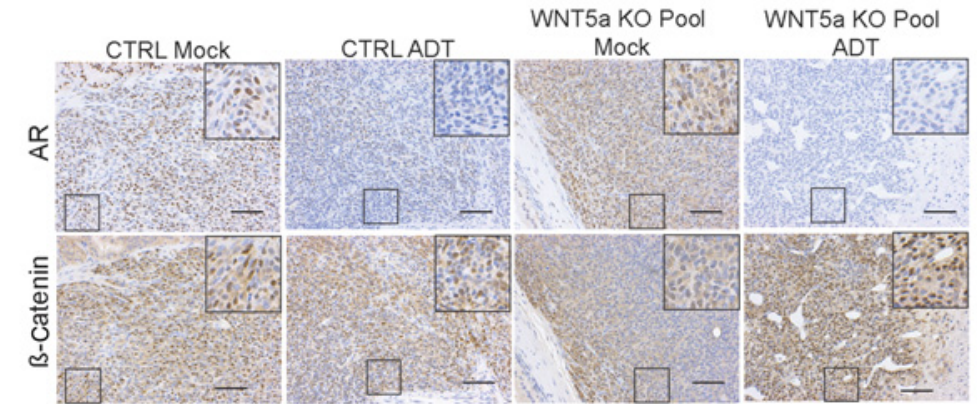

Figure 6.

WNT5a regulates c-Myc and AR expression. A-C, Representative immunoblots (A and $\mathbf{C}$ ) and AR mRNA quantification (B) in WNT5a KO CP2 cells. D, AR mRNA quantification in CP2 cells treated as indicated. E, mRNA quantification in WNT5a KO CP2 cells. F, mRNA quantification in WNT5a KO CP2 cells treated with siCTRL. G, CP2 orthograft volumes of Mock- and ADT-treated mice. H, Representative IHC images of CP2 prostate orthograft sections. I-J, Representative immunoblots (I) and growth rates (J) in active WNT5a-expressing C4-2 cells treated with siAR. K, Schematic showing the role of WNT5a in mutant Ctnnb7-driven murine prostate cancer. Unpaired two-tailed Student $t$ test for $\mathbf{E}$ and $\mathbf{F}$ and ANOVA Tukey test for $\mathbf{B}, \mathbf{D}, \mathbf{G}$, and $\mathbf{J}$. ${ }^{*}, P<0.05$. 


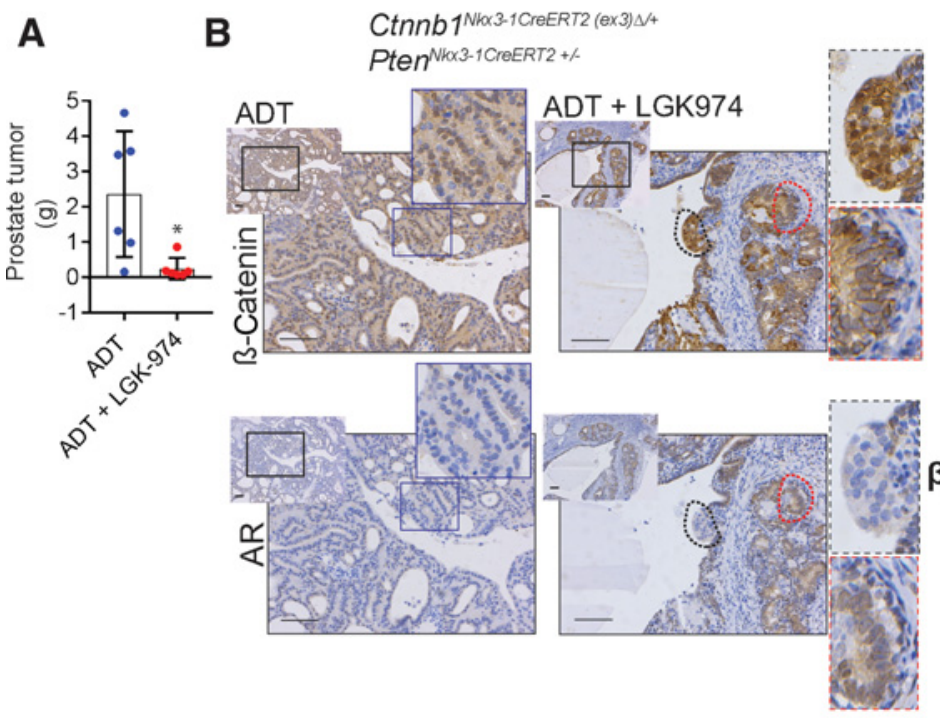

D

Murine model signature

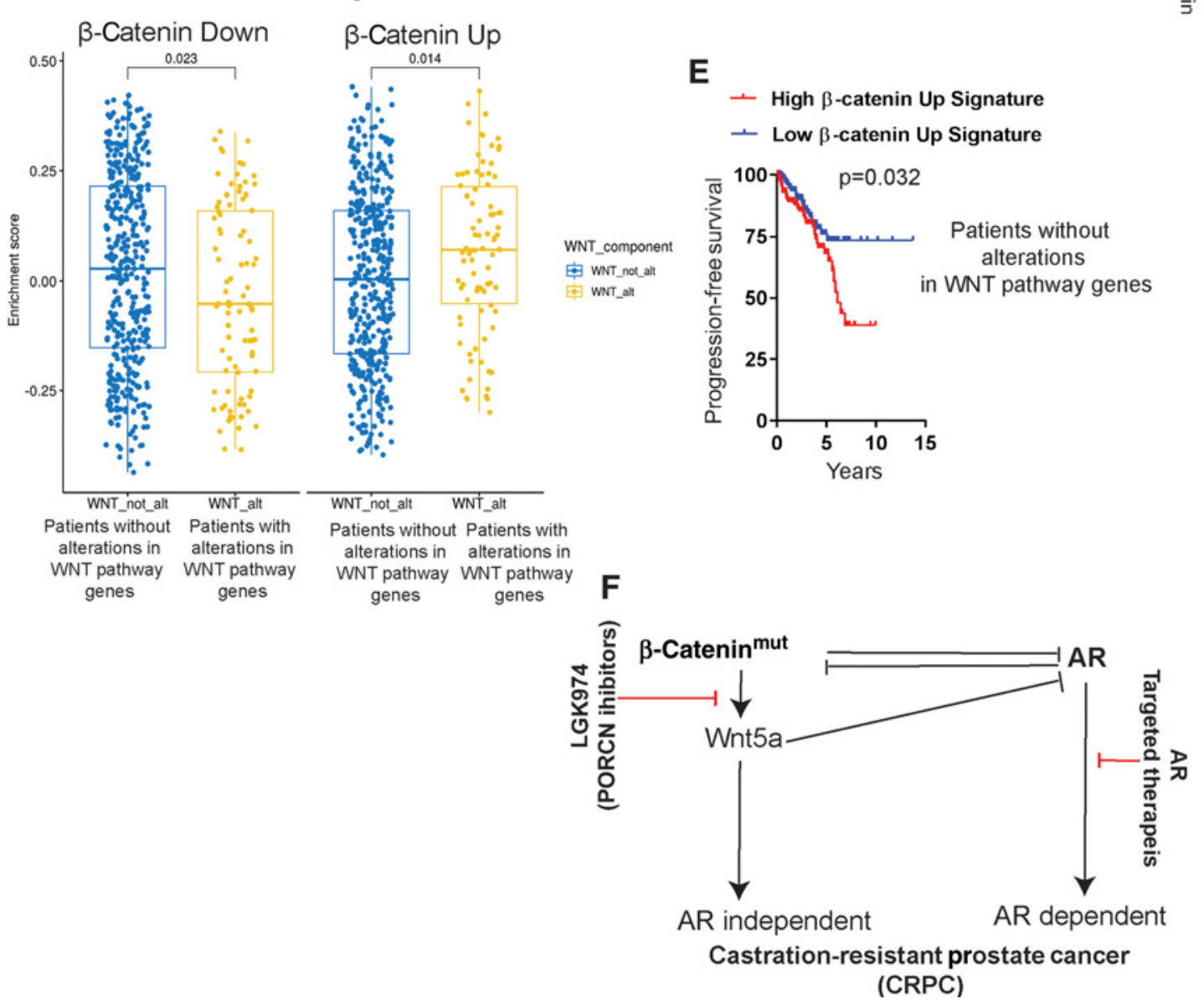

Figure 7.

Dual targeting of AR and WNT signaling in Ctnnb1-mutant prostate cancer. A, Prostate tumor weights from Ctnnb7 $7^{N k \times 3-1 C r e E R T(e \times 3) \Delta /+P t e n^{N k} \times 3-1 C r e E R T 2+/-}$ mice. Unpaired two-tailed Student $t$ test; *, $P<0.05$. B, Representative immunostained images of prostate sections. C, GSEA of $B$-catenin up and down signatures in patients with WNT pathway alterations. D, Gene set variation analysis enrichment per patient (TCGA prostate cancer dataset) of $B$-catenin up and down signatures (two-sided Wilcoxon rank-sum test). E, Kaplan-Meier plot showing adenocarcinoma cases without WNT pathway alterations with high B-catenin up (above median; $n=200$ ) and low $B$-catenin up (below median; $n=200$ ) gene set variation analysis enrichment scores. F, Schematic showing mutual antagonism between $B$-catenin and AR signaling in prostate cancer progression. 
availability. Overall, dual targeting of AR and WNT signaling may be required for treating prostate tumors with $ß$-catenin activation.

To investigate the clinical relevance of our models, we generated ß-catenin-upregulated and-downregulated genesets (Supplementary Fig. S7C and S7D). Consistent with the importance of WNT5a in sustaining the growth of Ctnnb1-mutant tumors, WNT5a is a central player associated with all top ten pathways of the B-catenin Up geneset (Supplementary Fig. S7E). Patients with prostate cancer with WNT pathway genes alterations showed higher enrichment for $B$-catenin Up signature and lower enrichment of $ß$-catenin Down signature compared with patients without alterations (Fig. 7C). Furthermore, by performing enrichment analyses per patient, we confirm that patients with WNT pathway genes alterations have significantly lower or higher enrichment for $B$-catenin Down or Up signature, respectively, compared with patients without alterations (Fig. 7D). Because tumor microenvironment can secrete WNT ligands (32), we investigated the prognostic value of $B$-catenin Up signature in the patients with prostate cancer without WNT pathway gene alterations. Patients with high (above median) enrichments for $B$-catenin Up signature have significantly poorer prognosis compared with those with low (below median) enrichments (Fig. 7E).

Patients with prostate cancer with WNT pathway gene alterations have a poor prognosis. Our murine models of mutant Ctnnb1-driven prostate cancer are representative of prostate cancers with WNT signaling gene alterations that may lead to B-catenin activation. Importantly, the AR pathway and $B$-catenin activation exhibit mutual antagonism. Prostate tumors with activated $\beta$-catenin progress to ARindependent CRPCs. WNT5a plays a central role in sustaining the growth of $B$-catenin-driven tumors and repressing AR expression. Highlighting the reciprocal regulation, AR inhibits WNT5a expression and noncanonical functions of WNT5a. Hence, dual targeting of AR signaling and inhibition of WNT secretion may represent a potential therapeutic strategy to treat prostate cancers with WNT pathway gene alterations (Fig. 7F).

\section{Discussion}

A better understanding of AR bypass pathways may facilitate new treatment combinations to tackle AR-independent mCRPC progression (33). Patients with prostate cancer with genomic alterations in WNT pathway (leading to ß-catenin activation) have a poor prognosis (4). Using murine models of mutant Ctnnb1-driven prostate cancer, we show that $B$-catenin-mutant tumors are refractory to ADT and develop AR-independent mCRPC. Mechanistically, activated Bcatenin-induced WNT5a governs the growth of Ctnnb1-mutant tumors and progression to AR-independent CRPC. Our work highlights the antagonistic cross-talk between WNT/B-catenin and AR signaling and raises the possibility of dual targeting of AR and WNT/Bcatenin signaling in the treatment of prostate cancers with $B$-catenin activation.

The contribution of $B$-catenin activation in prostate cancer progression is poorly understood. The oncogenic B-catenin activation cooperates with Pten loss to decrease the latency of prostate cancer progression. Almost half of the prostate cancer cases with the CTNNB1 mutation have associated PTEN haploinsufficiency (34). Interestingly, ß-catenin-mutant prostate tumors with Pten haploinsufficiency exhibit further loss of Pten, mimicking Pten-null ß-catenin-mutant tumors. Furthermore, $B$-catenin inversely associates with PTEN levels in clinical prostate cancer.

Both basal and luminal cells of prostate epithelium can harbor oncogenic mutations and become the cell of origin in prostate cancer (35-37). ß-Catenin activation may affect cancer progression differently in different cells. To ascertain the role of B-catenin activation in disease progression, we targeted the oncogenic Ctnnb1 mutation in both basal and luminal prostate epithelial cells using both Probasin and inducible Nkx3.1 Cre. Both basal and luminalderived prostate tumors show cooperation of Ctnnb1 mutation with Pten deletion, with similar cancer progression, but metastases were evident only in mice with Probasin-cre-driven tumors.

An important question that remains in the field of prostate cancer biology is whether AR and $B$-catenin pathways cooperate or oppose each other $(38,39)$. We show that mutant $\beta$-catenin inhibits the canonical AR functions, and the Ctnnb1-mutant prostate tumors have pseudo-castration transcriptomic signature. Earlier work on Ctnnb1driven prostate cancer has implicated a confounding role for AR in oncogenic B-catenin-driven prostate tumorigenesis (38). We show that under castrated conditions, Ctnnb1 mutation cooperated with Pten loss to drive AR-independent luminal-derived prostate cancer. Our work highlights the functional cooperation between $\mathrm{B}$-catenin activation and Pten deletion in driving AR-independent CRPC, independent of the cell of origin.

The noncanonical WNTs, such as WNT5a, may also govern Bcatenin-independent pathways such as $\mathrm{Ca}^{2+}$ signaling (9). WNT5a is one of the most enriched WNTs in mutant Ctnnb1-driven CRPC and metastases. Specifically, expression of $B$-catenin target genes such as $c-$ Myc required WNT5a. Mechanistically, WNT5a sustains c-Myc expression by regulating nuclear localization of $B$-catenin (even the mutant form). Further insights into how WNT5a may mediate nuclear localization of mutant $B$-catenin may uncover regulatory components of WNT/B-catenin signaling beyond destruction complex. As previously shown, Axin2 induced in the absence of WNT5a may also decrease the expression of c-Myc (40). Our work implicates a metabolic role of WNT5a in Ctnnb1-mutant cells by sustaining the expression of c-Myc and HK2 $(30,41,42)$. Thus, the mutant Ctnnb1-driven glucose metabolism may support the growth of AR-independent CRPC.

Interestingly, in Ctnnb1-mutant cells, WNT5a repressed AR expression via NFkBp65. Importantly, WNT5a sustained the growth of AR pathway-sensitive human CRPC C4-2 cells in the absence of AR. WNT5a was sufficient to induce c-Myc expression and increase the growth of AR-deficient PC3M human prostate cancer cells. WNT5a expression is associated with poor progression-free survival in ADTtreated patients with prostate cancer. Analysis of circulating tumor cells from patients with prostate cancer highlights the prevalence of WNT5a in resistance to AR-targeted therapy (43-45). In addition to altering the expression $B$-catenin target genes including WNT5a, AR also inhibited WNT5a-driven noncanonical CAMKII activation.

RSPO2, commonly amplified in prostate cancer, when bound to its receptors LGR5 or LGR6, amplifies WNT signaling. We observe that WNT5a sustains the expression of LGR5 and LGR6 by repressing AR expression in $\beta$-catenin-mutant prostate tumors. Thus, by decreasing AR expression, WNT5a may maintain the WNT/B-catenin signaling.

In this study, we show mutual antagonism between activated $B$-catenin and AR signaling in murine prostate cancers. Tumors with B-catenin activation depend on WNT signaling to sustain tumor growth, especially under ADT. The noncanonical WNT ligands such as WNT5a can exert their effects through multiple pathways (including $ß$-catenin-independent function). LGK974, a porcupine (PORCN) inhibitor in clinical trials (46), suppresses WNT signaling by blocking the secretion of WNTs. Combined ADT and LGK974 treatment was effective in regressing Ctnnb1-mutant tumors. However, even in the regressed prostates, the prostatic epithelium showed varied 
enrichment of cells with prominent nuclear $ß$-catenin indicative of persistent Ctnnb1-mutant cancer cells. Hence, continuous treatment with PORCN inhibitors may be required to restrain the growth of $ß$-catenin-driven prostate tumors.

Overall, our work indicates the potential use of $ß$-catenin-induced geneset signature derived from murine models in risk stratification for progression to CRPC in addition to the alterations in WNT pathway genes. Based on the reciprocal regulation of WNT/B-catenin and AR signaling, we propose dual targeting of $\mathrm{AR}$ and WNT signaling in patients with prostate cancer with genetic alterations in the WNT pathway.

\section{Disclosure of Potential Conflicts of Interest}

O.J. Sansom reports receiving commercial research grant from AstraZeneca and Novartis. No potential conflicts of interest were disclosed by the other authors.

\section{Authors' Contributions}

Conception and design: R. Patel, E.A. Brzezinska, O.J. Sansom, H.Y. Leung Development of methodology: R. Patel, E.A. Brzezinska, H.Y. Leung Acquisition of data (provided animals, acquired and managed patients, provided facilities, etc.): R. Patel, E.A. Brzezinska, I. Ahmad, E. Mui, M. Gao, V. Harle, E.H. Tan, G. Malviya, A. Mrowinska, L.K. Rushworth, G. Mackay

\section{References}

1. Yap TA, Smith AD, Ferraldeschi R, Al-Lazikani B, Workman P, de Bono JS. Drug discovery in advanced prostate cancer: translating biology into therapy. Nat Rev Drug Discovery 2016;15:699-718.

2. Watson PA, Arora VK, Sawyers CL. Emerging mechanisms of resistance to androgen receptor inhibitors in prostate cancer. Nat Rev Cancer 2015, 15:701.

3. Bluemn EG, Coleman IM, Lucas JM, Coleman RT, Hernandez-Lopez S, Tharakan R, et al. Androgen receptor pathway-independent prostate cancer is sustained through FGF signaling. Cancer Cell 2017;32:474-89.

4. Robinson D, Van Allen EM, Wu YM, Schultz N, Lonigro RJ, Mosquera JM, et al. Integrative clinical genomics of advanced prostate cancer. Cell 2015;161:1215 28.

5. Wyatt AW, Azad AA, Volik SV, Annala M, Beja K, McConeghy B, et al. Genomic alterations in cell-free DNA and enzalutamide resistance in castration-resistant prostate cancer. JAMA Oncol 2016;2:1598-606.

6. Armenia J, Wankowicz SAM, Liu D, Gao J, Kundra R, Reznik E, et al. The long tail of oncogenic drivers in prostate cancer. Nat Genet 2018;50:645-51.

7. Clevers H, Nusse R. Wnt/beta-catenin signaling and disease. Cell 2012;149: 1192-205.

8. Yochum GS, Sherrick CM, Macpartlin M, Goodman RH. A beta-catenin/TCFcoordinated chromatin loop at MYC integrates $5^{\prime}$ and $3^{\prime}$ Wnt responsive enhancers. PNAS 2010;107:145-50.

9. Grumolato L, Liu G, Mong P, Mudbhary R, Biswas R, Arroyave R, et al. Canonical and noncanonical Wnts use a common mechanism to activate completely unrelated coreceptors. Genes Dev 2010;24:2517-30.

10. Mikels AJ, Nusse R. Purified Wnt5a protein activates or inhibits beta-cateninTCF signaling depending on receptor context. PLoS Biol 2006;4:e115.

11. Murillo-Garzón V, Kypta R. WNT signalling in prostate cancer. Nat Rev Urol 2017;14:683.

12. Jefferies MT, Cox AC, Shorning BY, Meniel V, Griffiths D, Kynaston HG, et al PTEN loss and activation of K-RAS and beta-catenin cooperate to accelerate prostate tumourigenesis. J Pathol 2017;243:442-56.

13. Francis JC, Thomsen MK, Taketo MM, Swain A. beta-catenin is required for prostate development and cooperates with Pten loss to drive invasive carcinoma. PLos Genet 2013;9:e1003180.

14. Ahmad I, Patel R, Singh LB, Nixon C, Seywright M, Barnetson RJ, et al. HER2 overcomes PTEN (loss)-induced senescence to cause aggressive prostate cancer. PNAS 2011;108:16392-7.

15. Cancer Genome Atlas Research N. The molecular taxonomy of primary prostate cancer. Cell 2015;163:1011-25.

16. Cerami E, Gao J, Dogrusoz U, Gross BE, Sumer SO, Aksoy BA, et al. The cBio cancer genomics portal: an open platform for exploring multidimensional cancer genomics data. Cancer Discov 2012;2:401-4.
Analysis and interpretation of data (e.g., statistical analysis, biostatistics, computational analysis): R. Patel, E.A. Brzezinska, P. Repiscak, V. Harle, G. Malviya, A. Mrowinska, J. Edwards, C. Ntala, A. Hedley, S. Tardito, H.Y. Leung Writing, review, and/or revision of the manuscript: R. Patel, P. Repiscak, I. Ahmad, M. Gao, C.J. Loveridge, S. Tardito, O.J. Sansom, H.Y. Leung

Administrative, technical, or material support (i.e., reporting or organizing data, constructing databases): R. Patel, A. Blomme, C.J. Loveridge, C. Nixon Study supervision: R. Patel, O.J. Sansom, H.Y. Leung

Other (assisted with the tissue-based work and provided the tissue and linked clinical data): J. Edwards

\section{Acknowledgments}

We thank Beatson Institute's core research, biological, and histologic services. This work was supported by Cancer Research UK (A15151, A10419, and A17196), Prostate Cancer UK (PG10-10), and Prostate Cancer Foundation Award. We thank Joel Johansson for discussions.

The costs of publication of this article were defrayed in part by the payment of page charges. This article must therefore be hereby marked advertisement in accordance with 18 U.S.C. Section 1734 solely to indicate this fact.

Received May 31, 2019; revised September 4, 2019; accepted November 8, 2019; published first November 12, 2019

17. Gao J, Aksoy BA, Dogrusoz U, Dresdner G, Gross B, Sumer SO, et al. Integrative analysis of complex cancer genomics and clinical profiles using the cBioPortal. Sci Signal 2013;6:pl1.

18. Wu X, Wu J, Huang J, Powell WC, Zhang J, Matusik RJ, et al. Generation of a prostate epithelial cell-specific Cre transgenic mouse model for tissue-specific gene ablation. Mech Dev 2001;101:61-9.

19. Suzuki A, Yamaguchi MT, Ohteki T, Sasaki T, Kaisho T, Kimura Y, et al. T cellspecific loss of Pten leads to defects in central and peripheral tolerance. Immunity 2001;14:523-34

20. Harada N, Tamai Y, Ishikawa T, Sauer B, Takaku K, Oshima M, et al. Intestinal polyposis in mice with a dominant stable mutation of the beta-catenin gene. EMBO J 1999;18:5931-42.

21. Wang X, Kruithof-de Julio M, Economides KD, Walker D, Yu H, Halili MV, et al. A luminal epithelial stem cell that is a cell of origin for prostate cancer. Nature 2009;461:495-500

22. Luche H, Weber O, Nageswara Rao T, Blum C, Fehling HJ. Faithful activation of an extra-bright red fluorescent protein in "knock-in" Cre-reporter mice ideally suited for lineage tracing studies. Eur J Immunol 2007;37:43-53.

23. Patel R, Fleming J, Mui E, Loveridge C, Repiscak P, Blomme A, et al. Sprouty2 loss-induced IL6 drives castration-resistant prostate cancer through scavenger receptor B1. EMBO Mol Med 2018;10. DOI: 10.15252/emmm.201708347.

24. Kozlowski JM, Fidler IJ, Campbell D, Xu ZL, Kaighn ME, Hart IR. Metastatic behavior of human tumor cell lines grown in the nude mouse. Cancer Res 1984 44:3522-9.

25. Lukacs RU, Goldstein AS, Lawson DA, Cheng D, Witte ON. Isolation, cultivation and characterization of adult murine prostate stem cells. Nat Protoc 2010;5:702-13.

26. Patel R, Gao M, Ahmad I, Fleming J, Singh LB, Rai TS, et al. Sprouty2, PTEN, and PP2A interact to regulate prostate cancer progression. J Clin Invest 2013;123 $1157-75$

27. Carver BS, Chapinski C, Wongvipat J, Hieronymus H, Chen Y, Chandarlapaty S, et al. Reciprocal feedback regulation of PI3K and androgen receptor signaling in PTEN-deficient prostate cancer. Cancer Cell 2011;19:575-86.

28. van Amerongen R, Fuerer C, Mizutani M, Nusse R. Wnt5a can both activate and repress Wnt/beta-catenin signaling during mouse embryonic development Dev Biol 2012;369:101-14

29. Sansom OJ, Meniel VS, Muncan V, Phesse TJ, Wilkins JA, Reed KR, et al. Myc deletion rescues Apc deficiency in the small intestine. Nature 2007;446:676-9.

30. Stine ZE, Walton ZE, Altman BJ, Hsieh AL, Dang CV. MYC, metabolism, and cancer. Cancer Discov 2015;5:1024-39.

31. Bradley EW, Drissi MH.WNT5A regulates chondrocyte differentiation through differential use of the CaN/NFAT and IKK/NF- $\kappa B$ Pathways. Mol Endocrinol 2010;24:1581-93. 


\section{Patel et al.}

32. Nitzki F, Zibat A, Konig S, Wijgerde M, Rosenberger A, Brembeck FH, et al. Tumor stroma-derived Wnt5a induces differentiation of basal cell carcinoma of Ptch-mutant mice via CaMKII. Cancer Res 2010;70:2739-48.

33. Brennen $\mathrm{WN}$, Isaacs JT. Cellular origin of androgen receptor pathwayindependent prostate cancer and implications for therapy. Cancer Cell 2017; 32:399-401.

34. Gillard M, Lack J, Pontier A, Gandla D, Hatcher D, Sowalsky AG, et al. Integrative genomic analysis of coincident cancer foci implicates CTNNB1 and PTEN alterations in ductal prostate cancer. Eur Urol Focus 2019;5:433-42.

35. Wang ZA, Mitrofanova A, Bergren SK, Abate-Shen C, Cardiff RD, Califano A et al. Lineage analysis of basal epithelial cells reveals their unexpected plasticity and supports a cell-of-origin model for prostate cancer heterogeneity. Nat Cell Biol 2013;15:274-83.

36. Smith BA, Sokolov A, Uzunangelov V, Baertsch R, Newton Y, Graim K, et al. A basal stem cell signature identifies aggressive prostate cancer phenotypes. PNAS 2015;112:E6544-52.

37. Lu TL, Chen CM. Differential requirements for beta-catenin in murine prostate cancer originating from basal versus luminal cells. J Pathol 2015;236:290-301.

38. Lee SH, Luong R, Johnson DT, Cunha GR, Rivina L, Gonzalgo ML, et al Androgen signaling is a confounding factor for beta-catenin-mediated prostate tumorigenesis. Oncogene 2016;35:702-14.
39. Chesire DR, Isaacs WB. Ligand-dependent inhibition of beta-catenin/TCF signaling by androgen receptor. Oncogene 2002;21:8453-69.

40. Rennoll SA, Konsavage WM Jr., Yochum GS. Nuclear AXIN2 represses MYC gene expression. Biochem Biophys Res Commun 2014;443:217-22.

41. Bernard D, Pourtier-Manzanedo A, Gil J, Beach DH. Myc confers androgen-independent prostate cancer cell growth. J Clin Invest 2003, 112:1724-31.

42. Massie CE, Lynch A, Ramos-Montoya A, Boren J, Stark R, Fazli L, et al. The androgen receptor fuels prostate cancer by regulating central metabolism and biosynthesis. EMBO J 2011;30:2719-33.

43. Miyamoto DT, Zheng Y, Wittner BS, Lee RJ, Zhu H, Broderick KT, et al. RNASeq of single prostate CTCs implicates noncanonical Wnt signaling in antiandrogen resistance. Science 2015;349:1351-6.

44. Singhal U, Wang Y, Henderson J, Niknafs YS, Qiao Y, Gursky A, et al. Multigene profiling of CTCs in mCRPC identifies a clinically relevant prognostic signature. Mol Cancer Res 2018;16:643-54.

45. Lee GT, Kwon SJ, Kim J, Kwon YS, Lee N, Hong JH, et al. WNT5A induces castration-resistant prostate cancer via CCL2 and tumour-infiltrating macrophages. Br J Cancer 2018;118:670-8.

46. Liu J, Pan S, Hsieh MH, Ng N, Sun F, Wang T, et al. Targeting Wnt-driven cancer through the inhibition of Porcupine by LGK974. PNAS 2013;110: 20224-9. 


\section{Cancer Research}

$A A C R$ 年erican Assciation

The Journal of Cancer Research (1916-1930) | The American Journal of Cancer (1931-1940)

\section{Activation of $\beta$-Catenin Cooperates with Loss of Pten to Drive AR-Independent Castration-Resistant Prostate Cancer}

Rachana Patel, Elspeth A. Brzezinska, Peter Repiscak, et al.

Cancer Res 2020;80:576-590. Published OnlineFirst November 12, 2019.

Updated version Access the most recent version of this article at: doi:10.1158/0008-5472.CAN-19-1684

Supplementary Access the most recent supplemental material at:

Material http://cancerres.aacrjournals.org/content/suppl/2019/11/12/0008-5472.CAN-19-1684.DC1

Cited articles This article cites 44 articles, 14 of which you can access for free at:

http://cancerres.aacrjournals.org/content/80/3/576.full\#ref-list-1

E-mail alerts Sign up to receive free email-alerts related to this article or journal.

Reprints and To order reprints of this article or to subscribe to the journal, contact the AACR Publications Department at Subscriptions pubs@aacr.org.

Permissions To request permission to re-use all or part of this article, use this link http://cancerres.aacrjournals.org/content/80/3/576.

Click on "Request Permissions" which will take you to the Copyright Clearance Center's (CCC) Rightslink site. 